\title{
LARGE-TIME OPTION PRICING USING THE DONSKER-VARADHAN LDP-CORRELATED STOCHASTIC VOLATILITY WITH STOCHASTIC INTEREST RATES AND JUMPS
}

\author{
BY MARTIN FORDE AND ROHINI KUMAR ${ }^{1}$ \\ King's College London and Wayne State University
}

\begin{abstract}
We establish a large-time large deviation principle (LDP) for a general mean-reverting stochastic volatility model with nonzero correlation and sublinear growth for the volatility coefficient, using the Donsker-Varadhan [Comm. Pure Appl. Math. 36 (1983) 183-212] LDP for the occupation measure of a Feller process under mild ergodicity conditions. We verify that these conditions are satisfied when the process driving the volatility is an OrnsteinUhlenbeck (OU) process with a perturbed (sublinear) drift. We then translate these results into large-time asymptotics for call options and implied volatility and we verify our results numerically using Monte Carlo simulation. Finally, we extend our analysis to include a CIR short rate process and an independent driving Lévy process.
\end{abstract}

1. Introduction. The last few years has seen the emergence of a number of articles on large-time asymptotics for stochastic volatility models, with and without jumps. Using the Gärtner-Ellis theorem from large deviations theory, [13] compute the asymptotic (i.e., leading order) implied volatility smile for the wellknown Heston model in the so-called large-time, large log-moneyness regime, under a mild restriction on the model parameters, and the rate function is computed numerically as a Fenchel-Legendre transform which is just a one-dimensional root-finding exercise. [17] show that the asymptotic smile can actually be computed in closed-form via the SVI parameterization and [14] compute the correction term to this smile using saddlepoint methods; [12] derives a similar result for the Stein-Stein model and [19] derive a similar result for a displaced Heston model (and relax the aforementioned condition on the parameters). [18] extended the results in [13] to a general class of affine stochastic volatility models (with jumps), which includes the Heston, Bates and the Barndorff-Nielsen-Shephard model, and under mild assumptions, they show that the limiting smile necessarily corresponds to the smile generated by an exponential Lévy model. More recently, [15] compute large-time asymptotics for a fractional local-stochastic volatility model and large-time asymptotics for European and barrier options under conventional and

Received November 2014; revised February 2016.

${ }^{1}$ Supported by NSF Grant DMS-12-09363.

MSC2010 subject classifications. 60G99, 60J60, 60J25.

Key words and phrases. Stochastic volatility, large deviations, Donsker-Varadhan large deviation principle, implied volatility asymptotics, ergodic processes, occupation measures. 
fractional exponential Lévy models, using the deAcosta LDP for a Lévy process on path space.

[11] derives a large deviation principle for the log stock price under an uncorrelated stochastic volatility model driven by an Ornstein-Uhlenbeck process with a bounded volatility function. For this, we use the fact that the occupation measure for the Ornstein-Uhlenbeck process satisfies an LDP with a good, convex lower semicontinuous rate function under the topology of weak convergence (and under the Prokhorov metric); see Section 7 in Donsker and Varadhan [5] (see also page 178 in Stroock [25] and Proposition 1.3 in [21]), combined with the standard contraction principle and exponential tightness. The large-time regime is also closely related to the small-time, fast mean reverting regime considered in Feng, Fouque and Kumar [8] for a more general stochastic volatility model. The problem then falls into the class of homogenization and averaging problems for nonlinear HJB type equations, where the fast volatility variable lives on a noncompact set.

1.1. Outline of article. In this article, we consider a stochastic volatility model for a log stock price process $X_{t}$ of the form

$$
\left\{\begin{array}{l}
d X_{t}=-\frac{1}{2} \sigma\left(Y_{t}\right)^{2} d t+\sigma\left(Y_{t}\right)\left(\sqrt{1-\rho^{2}} d W_{t}^{1}+\rho d W_{t}^{2}\right), \\
d Y_{t}=\left(-\alpha Y_{t}+g\left(Y_{t}\right)\right) d t+d W_{t}^{2},
\end{array}\right.
$$

where $W^{1}, W^{2}$ are independent Brownian motions. We first relax the assumptions that $\sigma$ is bounded and $\rho$ and $g$ are zero that are imposed in [11]. This requires an auxiliary result, namely that the variance of a probability measure on the real line can be bounded in terms of the Donsker-Varadhan rate function of the measure. Using this property, we then establish an LDP for $\left(X_{t} / t\right)$ using the trivial joint LDP for the two independent variables $\left(W_{t}^{1} / t, \mu_{t}\right)$ (where $\mu_{t}$ is the occupation measure of $Y$ ), combined with the extended contraction principle for noncontinuous functionals given in Theorem 4.2.23 in [3]. This is the same theorem which can be used to prove the Freidlin-Wentzell small-noise LDP from Schilder's theorem, despite the lack of continuity of the Itô map in the sup norm topology (see, e.g., proof of Theorem 5.6.7 in [3]), and is also used in rough paths theory to prove the smallnoise LDP for a rough differential equation driven by fractional Brownian motion (cf. Section 15.7 and Proposition 19.14 in [16]). The rate function for $X_{t} / t$ in this article has the variational representation $I(x)=\inf _{\mu \in \mathcal{P}(\mathbb{R})}\left[\frac{(x-M(\mu))^{2}}{2 v(\mu)}+I_{Y}(\mu)\right]$, for some linear functionals $M(\mu), v(\mu)$ which depend on the correlation $\rho$ between the $\log$ stock price process and the $Y$ process. For the at-the-money case $x=0$ with $\rho=0$, we find that the rate function reduces to the classical Rayleigh-Ritz formula for the principal eigenvalue $\lambda_{1}$ of an associated Sturm-Liouville equation.

In Section 5, we translate these results into large-time asymptotics for call options and implied volatility; this requires computing the corresponding LDP for the log stock price under the so-called Share measure $\mathbb{P}^{*}$ associated with using 
the stock price process as the numéraire, and in Section 6 , we compute $I(x)$ numerically, using the Ritz method from the theory of calculus of variations. The Ritz method is described at length in Gelfand and Fomin [10]-we choose an $n$ dimensional subspace of the space of admissible functions, in this case the Hilbert space $L^{2}\left(\mu_{\infty}\right)$, and we then minimize the objective function $\frac{(x-M(\mu))^{2}}{2 v(\mu)}+I_{Y}(\mu)$ by minimizing over the subspace for the $n$ Fourier coefficients.

In Section 7, we enrich the general model with an additional independent CIR short rate process $r_{t}$ and an independent driving Lévy process $Z_{t}$. It is well known that stochastic interest rates make a significant difference to the price of European options at large maturities, but to our knowledge this effect has never been properly quantified using asymptotics; specifically, we show that the log stock price now satisfies the LDP with rate function $I_{r}(x)=\inf _{a, y, z: a+y+z=x}\left[I(y)+I_{\mathrm{CIR}}(a)+\right.$ $\left.V_{J}^{*}(z)\right]$ where $I_{\mathrm{CIR}}(a)=\kappa_{r}^{2}\left(a-\theta_{r}\right)^{2} /\left(2 a \sigma_{r}^{2}\right)$ is the rate function for $\frac{1}{t} \int_{0}^{t} r_{s} d s$, and $V_{J}^{*}(x)$ is the rate function for $Z_{t} / t$.

2. The Donsker-Varadhan large deviation principle. Let $\Omega$ denote the space of real-valued functions $\omega(\cdot)$ on $-\infty<t<\infty$ with discontinuities of the first kind, normalized to be right continuous, and with convergence induced by the Skorokhod topology on bounded intervals. Let $\left(Y, \mathbb{P}_{y}\right)$ be a Markov process on $\Omega$ with invariant distribution $\mu_{\infty}(d y)$ such that the mapping $y \rightarrow \mathbb{P}_{y}$ is weakly continuous (which implies the Feller property for the process $Y$ ). Let $p(t, x, d y$ ) denote the transition probability for $Y, P_{t}$ denote the semigroup associated with $Y$, and let $L$ denote the infinitesimal generator of $P_{t}$ and $\mathcal{D}=\mathcal{D}(L) \subset C_{b}(\mathbb{R})$ its domain. For each $t>0$ and $A \in \mathcal{B}(\mathbb{R})$, let

$$
\mu_{t}(A)=\frac{1}{t} \int_{0}^{t} 1_{A}\left(Y_{S}\right) d s
$$

denote the occupation time distribution of $Y$, that is, the proportion of time that $Y$ spends in the set $A$. For each $t>0$ and $\omega, \mu_{t}(\cdot)$ is a probability measure on $\mathbb{R}$. Let $\mathcal{P}(\mathbb{R})$ denote the space of probability measures on $(\mathbb{R}, \mathcal{B}(\mathbb{R})$ ). Then from [5] (or page 178 in Stroock [25] or Pinsky [23]), under suitable recurrence and transitivity conditions (see the next subsection for details), $\mu_{t}(\cdot)$ satisfies the LDP as $t \rightarrow \infty$ in the topology of weak convergence, with a convex, lower semicontinuous rate function $I_{Y}: \mathcal{P}(\mathbb{R}) \mapsto[0, \infty]$ given by

$$
I_{Y}(\mu)=-\inf _{u \in \mathcal{D}^{+}} \int_{-\infty}^{\infty} \frac{L u}{u} d \mu
$$

for each $\mu \in \mathcal{P}(\mathbb{R})$, where $\mathcal{D}^{+}$is the set of $u$ in the domain $\mathcal{D}$ of $L$ with $u \geq \varepsilon>0$ for some $\varepsilon>0$. More precisely, if we define a probability measure $Q_{t, y}$ on $\mathcal{P}(\mathbb{R})$ by $Q_{t, y}=\mathbb{P}_{y} \circ \mu_{t}^{-1}$, then for any closed set $C \subset \mathcal{P}(\mathbb{R})$ (weak topology) and for 
any open set on $G \subset \mathcal{P}(\mathbb{R})$ we have

$$
\begin{aligned}
& \limsup _{t \rightarrow \infty} \frac{1}{t} \log Q_{t, y}(C) \leq-\inf _{\mu \in C} I_{Y}(\mu), \\
& \liminf _{t \rightarrow \infty} \frac{1}{t} \log Q_{t, y}(G) \geq-\inf _{\mu \in G} I_{Y}(\mu) .
\end{aligned}
$$

$I_{Y}(\cdot)$ is know as the $I$-function for the process $Y$.

REMARK 2.1. By the ergodic theorem, $Q_{t, y} \stackrel{w}{\rightarrow} \delta_{\mu_{\infty}}$ as $t \rightarrow \infty$, and it is well known that $I_{Y}(\mu)=0$ if and only if $\mu=\mu_{\infty}$ (see, e.g., the proof of Corollary 1.5 in [23]).

2.1. Sufficient conditions for the LDP upper and lower bounds. In [26] (page 34) and [5, 6], it is shown that the following five conditions imply the LDP upper bound in (2.2).

There exists a sequence $u_{n}$ of functions in $\mathcal{D}(L)$ with the five properties:

1. $u_{n}(y) \geq c>0$ for $y$ and $n$.

2. For all compact sets $K \subset \mathbb{R}$, there exists a constant $C_{K}$ such that $\sup _{y \in K} \sup _{n} u_{n}(y) \leq C_{K}$.

3. $V_{n}(y):=-\left(L u_{n} / u_{n}\right)(y) \geq-C$ for all $n$ and $y$.

4. There exists a function $V(y)$ such that for all $y \in \mathbb{R}, \lim _{n \rightarrow \infty} V_{n}(y)=V(y)$.

5. The set $\{y: V(y) \leq \ell\}$ is compact for all $\ell<\infty$.

Moreover, the following two conditions imply the LDP lower bound:

There exists a density function for $p(1, x, d y)$ with respect to a reference measure $\alpha$ on $\mathbb{R}$ such that:

I. $p(1, x, d y)=p(1, x, y) \alpha(d y)$.

II. $p(1, x, \cdot)$ as a mapping from $\mathbb{R} \rightarrow L^{1}(\alpha)$ is continuous.

These two conditions are given in $[6,26]$, where the LDP is proved as a corollary of a more general LDP on path space in terms of the entropy function (see Theorem 13.1.31 in [26]). These two conditions simplify the more cumbersome conditions for the LDP lower bound given on page 393 in [5].

\subsection{Examples: The $O U$ process and the perturbed $O U$ process.}

- For the Ornstein-Uhlenbeck process,

$$
d Y_{t}=-\alpha Y_{t} d t+d W_{t}
$$

conditions $1-5$ in Section 2.1 are satisfied with $u_{n}(y)=\cosh (n \theta(y / n))$, if $\theta(y)=y$ for $0 \leq y \leq 1$ and $\theta, \theta^{\prime}, \theta^{\prime \prime}$ are uniformly bounded on $\mathbb{R}$ and $\theta$ is odd (see Sections 7 in [5] and [6]), and in this case $V(y)=-\frac{L u}{u}(y)=$ 
$-\frac{1}{2}+y \alpha \tanh y$, which tends to $|y|$ as $y \rightarrow \pm \infty$. In the next bullet point, we will show that the two lower bounds are also satisfied, as a special case of a more general perturbed OU process. Thus, $\mu_{t}$ satisfies the LDP with rate $I_{Y}(\mu)$ as in (2.1) as $t \rightarrow \infty$. The OU process has a unique invariant distribution given by $\mu_{\infty}(y)=\left(\frac{\alpha}{\pi}\right)^{\frac{1}{2}} e^{-\alpha y^{2}}$, that is, $N\left(0, \frac{1}{2 \alpha}\right)$. If $\mu$ is absolutely continuous with respect to $\mu_{\infty}$, then [because the OU process is symmetric, that is, its generator is self-adjoint with respect to $\left.\mu_{\infty}(y)\right]$ we can simplify the rate function $I_{Y}$ to

$$
I_{Y}(\mu)=\frac{1}{2} \int_{-\infty}^{\infty} \psi^{\prime}(y)^{2} \mu_{\infty}(d y)
$$

if $\psi^{\prime} \in L^{2}\left(\mu_{\infty}\right)$, where $\phi=\frac{d \mu}{d \mu_{\infty}}$ is the Radon-Nikodým derivative and $\psi=$ $\sqrt{\phi}$ (see page 179 under exercise (8.28) in Stroock [25]). If $\mu$ is not absolutely continuous with respect to $\mu_{\infty}$, then $I_{Y}(\mu)=\infty$. The representation in (2.3) will be used for the numerics in Section 6 using the Ritz method.

- For a perturbed OU process of the form

$$
d Y_{t}=\left(-\alpha Y_{t}+g\left(Y_{t}\right)\right) d t+d W_{t},
$$

where $g$ is $C^{3}$ with sublinear growth at $\pm \infty$ and continuous bounded derivatives of all orders up and including 3 , the $-\alpha y$ drift term swamps the $g(y)$ term as $|y| \rightarrow \infty$ and the five conditions 1-5 for the LDP upper bound are still satisfied with the same $u_{n}(y)$ as above. This includes the case when, for example, $g(y)=$ $\alpha \theta$ for a constant $\theta$ which is the mean-reversion level for $Y$.

LEMMA 2.1. The perturbed OU process in (2.4) satisfies the two lower bound conditions I and II above.

Proof. See Appendix B.

We also note that the process $Y$ in (2.4) has a unique invariant distribution given by

$$
\mu_{\infty}(y)=\frac{e^{-\alpha y^{2}} e^{2 \int_{y_{0}}^{y} g(u) d u}}{\int_{-\infty}^{\infty} e^{-\alpha u^{2}} e^{2 \int_{y_{0}}^{u} g(v) d v d u} d u} .
$$

2.3. The Prokhorov metric on $\mathcal{P}(\mathbb{R})$ and goodness of the rate function $I_{Y}(\mu)$. We can also topologize $\mathcal{P}(\mathbb{R})$ with the Prokhorov metric, defined as

$$
d\left(\mu, \mu_{1}\right)=\inf \left\{\delta>0: \mu(C) \leq \mu_{1}\left(C^{\delta}\right)+\delta \text { for all closed } C \in \mathcal{B}(\mathbb{R})\right\}
$$

for $\mu, \mu_{1} \in \mathcal{P}(\mathbb{R})$, where $C^{\delta}$ is the $\delta$-neighbourhood of $C^{2}$ (see page 96 in Ethier and Kurtz [7]). Under this metric, $\mathcal{P}(\mathbb{R})$ is a metric space [note also that

\footnotetext{
${ }^{2}$ The set of all points which are of distance $\leq \delta$ from $C$.
} 
$d\left(\mu, \mu_{1}\right) \leq 1$ for all $\left.\mu, \mu_{1}\right]$. Moreover, $\mathbb{R}$ is separable, so convergence of measures in the Prokhorov metric is equivalent to weak convergence of measures (see Theorem 3.1 part (a) and part (b) in [7] for details), so the Donsker-Varadhan LDP for $\mu_{t}$ also holds in the topology induced by the metric $d$.

REMARK 2.2. By Lemma 7.1 (see also page 461) in [5] $\mu_{t}$ is exponentially tight in the weak topology (and thus also in the Prokhorov topology), and thus (by Lemma 1.2.18 in [3]) $I_{Y}(\cdot)$ is a $\operatorname{good}$ rate function.

2.4. The tail behaviour of probability measures inside the level sets of $I_{Y}$. The following lemma is the main observation on which the article is based, which characterizes the tail behaviour of the measures inside a level set of $I_{Y}$.

LEMMA 2.2. Consider the perturbed OU process in (2.4). Then for $\mu \in \mathcal{P}(\mathbb{R})$ we have the following bound for the second moment of $\mu$ in terms of $I_{Y}(\mu)$ :

$$
\int_{-\infty}^{\infty} y^{2} \mu(d y) \leq K_{2}(\alpha) I_{Y}(\mu)+K_{3}(\alpha)
$$

for some constants $K_{2}(\alpha)>0$ and $K_{3}(\alpha)$.

Proof. The infinitesimal generator $\mathcal{L}$ of $Y$ coincides with the differential operator $\mathcal{L}=(-\alpha y+g(y)) \partial_{y}+\frac{1}{2} \partial_{y y}^{2}$ on $C_{b}^{2}(\mathbb{R})$. Define a function $\psi$ such that

$$
\psi(y):= \begin{cases}y, & (0 \leq y \leq 1), \\ 2, & (y \geq 2), \\ \text { smoothly increasing, } & (1 \leq y \leq 2)\end{cases}
$$

and $\psi$ is an odd function. Consequently, $\psi, \psi^{\prime}, \psi^{\prime \prime}$ are uniformly bounded, $\psi^{\prime} \geq 0$ and $\psi(u) / u>0$ and is uniformly bounded when $u \neq 0$. Let $u_{n}(y)=e^{\frac{c}{2}\left[n \psi\left(\frac{y}{n}\right)\right]^{2}}$ with $c \in\left(0, \alpha \wedge\left(\frac{\alpha}{\sup _{u \neq 0} \frac{\psi(u)}{u} \psi^{\prime}(u)}\right)\right)$. Then

$$
\begin{aligned}
-L u_{n}(y)= & -\left[(-\alpha y+g(y))\left(\operatorname{cn} \psi\left(\frac{y}{n}\right) \psi^{\prime}\left(\frac{y}{n}\right)\right)\right. \\
& +\frac{1}{2}\left(c^{2} n^{2} \psi^{2}\left(\frac{y}{n}\right)\left(\psi^{\prime}\left(\frac{y}{n}\right)\right)^{2}+c\left(\psi^{\prime}\left(\frac{y}{n}\right)\right)^{2}\right. \\
& \left.\left.+c \psi\left(\frac{y}{n}\right) \psi^{\prime \prime}\left(\frac{y}{n}\right)\right)\right] 1_{\left\{\left|\frac{y}{n}\right| \leq 2\right\}} u_{n}(y) \\
=- & -\left[\left(-\alpha y^{2}+y g(y)\right)\left(c \frac{n}{y} \psi\left(\frac{y}{n}\right) \psi^{\prime}\left(\frac{y}{n}\right)\right)\right. \\
& +\frac{1}{2}\left(c^{2} y^{2} \frac{n^{2}}{y^{2}} \psi^{2}\left(\frac{y}{n}\right)\left(\psi^{\prime}\left(\frac{y}{n}\right)\right)^{2}+c\left(\psi^{\prime}\left(\frac{y}{n}\right)\right)^{2}\right.
\end{aligned}
$$




$$
\begin{aligned}
& \left.\left.+c \psi\left(\frac{y}{n}\right) \psi^{\prime \prime}\left(\frac{y}{n}\right)\right)\right] u_{n}(y) \\
-\frac{L u_{n}}{u_{n}}(y)= & c \frac{\psi(y / n)}{y / n} \psi^{\prime}(y / n)\left[\alpha y^{2}-y g(y)-\frac{c y^{2}}{2} \frac{\psi(y / n)}{y / n} \psi^{\prime}(y / n)\right] \\
& -\frac{1}{2}\left[c\left(\psi^{\prime}\left(\frac{y}{n}\right)\right)^{2}+c \psi\left(\frac{y}{n}\right) \psi^{\prime \prime}\left(\frac{y}{n}\right)\right] \\
= & I+I I .
\end{aligned}
$$

Observe that the second term II is uniformly bounded as $\psi, \psi^{\prime}, \psi^{\prime \prime}$ are uniformly bounded. As for the first term $I$, note that $\frac{\psi(u)}{u} \psi^{\prime}(u)>0$ and is uniformly bounded for $u \neq 0$, hence $-\frac{c y^{2}}{2} \frac{\psi(u)}{u} \psi^{\prime}(u)>-\frac{\alpha}{2} y^{2}$ if $c \frac{\psi(u) \psi^{\prime}(u)}{u}<\alpha$. Moreover, since $g(y)$ has sublinear growth, there exists a constant $c_{1}>0$ such that $\frac{\alpha}{2} y^{2}-y g(y)>-c_{1}$ for all $y$. Hence, $-\frac{L u_{n}}{u_{n}}(y)$ is uniformly bounded from below. Since $\psi(y / n)=$ $y / n$ and $\psi^{\prime}(y / n)=1$ for $|y| \leq n$, it is trivial to check that $-\frac{L u_{n}}{u_{n}}(y) \rightarrow(c \alpha-$ $\left.\frac{c^{2}}{2}\right) y^{2}-\operatorname{cyg}(y)-\frac{c}{2}$ pointwise as $n \rightarrow \infty$ and $L u_{n} \in C_{b}$ because $L u_{n}(y)=0$ for $y$ sufficiently large, so $u_{n} \in \mathcal{D}^{+}$. From this, we obtain

$$
I_{Y}(\mu)=\sup _{u \in \mathcal{D}^{+}}-\int_{-\infty}^{\infty} \frac{L u}{u} d \mu \geq-\int_{-\infty}^{\infty} \frac{L u_{n}}{u_{n}} d \mu .
$$

Taking the liminf of both sides as $n \rightarrow \infty$ and using Fatou's lemma, we obtain

$$
I_{Y}(\mu) \geq \liminf _{n \rightarrow \infty} \int_{-\infty}^{\infty}-\frac{L u_{n}}{u_{n}} d \mu \geq \int_{-\infty}^{\infty}\left(\left(c \alpha-\frac{c^{2}}{2}\right) y^{2}-\operatorname{cyg}(y)-\frac{c}{2}\right) \mu(d y) .
$$

Since $y g(y)$ is subquadratic, we can find a positive constant $K_{1}(\alpha)$ such that $(c \alpha-$ $\left.c^{2} / 2\right) y^{2}-\operatorname{cyg}(y)=\frac{\left(c \alpha-c^{2} / 2\right)}{2} y^{2}+\frac{\left(c \alpha-c^{2} / 2\right)}{2} y^{2}-\operatorname{cyg}(y) \geq \frac{\left(c \alpha-c^{2} / 2\right)}{2} y^{2}-K_{1}(\alpha)$. Thus, we have that $I_{Y}(\mu) \geq \frac{\left(c \alpha-c^{2} / 2\right)}{2} \int_{-\infty}^{\infty} y^{2} \mu(d y)-K_{1}(\alpha)-\frac{1}{2} c$, and the result follows by re-arranging.

3. The stochastic volatility model. We work on a probability space $(\Omega, \mathcal{F}, \mathbb{P})$ unless otherwise stated, with a filtration $\left(\mathcal{F}_{t}\right)_{t \geq 0}$ and satisfying the usual conditions. We consider the following stochastic volatility model for a log stock price process $X_{t}=\log S_{t}$ driven by a perturbed Ornstein-Uhlenbeck process $Y$ :

$$
\left\{\begin{array}{l}
d X_{t}=-\frac{1}{2} \sigma\left(Y_{t}\right)^{2} d t+\sigma\left(Y_{t}\right)\left(\rho d W_{t}^{2}+\bar{\rho} d W_{t}^{1}\right), \\
d Y_{t}=\left(-\alpha Y_{t}+g\left(Y_{t}\right)\right) d t+d W_{t}^{2}
\end{array}\right.
$$

where $\alpha>0, X_{0}=x_{0}, Y_{0}=y_{0}, W^{1}, W^{2}$ are two independent standard Brownian motions, $\rho \in(-1,1), \bar{\rho}=\sqrt{1-\rho^{2}}$ and we make the following assumptions on $\sigma$ and $g$ throughout. 
ASSUMPTION 3.1. $\quad \sigma: \mathbb{R} \mapsto(0, \infty)$ and $g: \mathbb{R} \mapsto \mathbb{R}$ are both continuous and satisfy the sublinear growth conditions ${ }^{3}$

$$
\sigma(y) \vee g(y) \leq K_{1}\left(1+|y|^{p}\right)
$$

for some $K_{1}>0, p \in(0,1)$.

ASSUMPTION 3.2. $g$ has continuous bounded derivatives of all orders order up and including 3 , and if $\rho \neq 0$ then $\sigma$ is differentiable and $\left|\sigma^{\prime}(y)\right|$ is bounded.

REMARK 3.3. Note that for the seemingly more general model:

$$
\left\{\begin{array}{l}
d X_{t}=-\frac{1}{2} f\left(V_{t}\right)^{2} d t+f\left(V_{t}\right)\left(\rho d W_{t}^{2}+\bar{\rho} d W_{t}^{1}\right), \\
d V_{t}=\left[\alpha\left(m-V_{t}\right)+\tilde{h}\left(V_{t}\right)\right] d t+\beta d W_{t}^{2}
\end{array}\right.
$$

for $\alpha, \beta>0$ and $f, \tilde{h}$ satisfying the same conditions as $\sigma$ and $g$ above, if we set $Y_{t}=\frac{1}{\beta}\left(V_{t}-m\right)$ and $\sigma(y)=f(\beta y+m), g(y)=\tilde{h}(\beta y+m)$, then we are transformed back to a model of the model in (3.1), so there is no loss of generality in our assumption of zero mean reversion level and vol-of-vol (i.e., diffusion coefficient) equal to 1 in the $Y$ process in (3.1).

We also set $S_{0}=1$ throughout (i.e., $x_{0}=0$ ) without loss of generality, because $X_{t}-x_{0}$ is independent of $x_{0}$ as the SDEs have no dependence on $x$.

3.1. The integrated variance. Now let $F: \mathcal{P}(\mathbb{R}) \mapsto \mathbb{R}^{+}$denote the linear functional defined by

$$
F(\mu)=\int_{-\infty}^{\infty} \sigma^{2}(y) \mu(d y) .
$$

Note that $F$ may not be continuous in the weak topology because $\sigma^{2}$ may not be bounded. Define

$$
F\left(\mu_{t}\right)=\int_{-\infty}^{\infty} \sigma^{2}(y) \mu_{t}(d y)=\frac{1}{t} \int_{0}^{t} \sigma^{2}\left(Y_{s}\right) d s,
$$

where $\mu_{t}(d y)$ is the occupation measure of $Y$; then we see that $F\left(\mu_{t}\right)$ is the timeaverage of the instantaneous variance for $Y$. We also define

$$
\bar{\sigma}^{2}=\int_{-\infty}^{\infty} \sigma^{2}(y) \mu_{\infty}(y) d y,
$$

where $\mu_{\infty}$ is defined in (2.5).

\footnotetext{
${ }^{3}$ The same condition appears in Feng, Fouque and Kumar [8].
} 


\section{Large-time asymptotics for the stochastic volatility model.}

4.1. The main result: The LDP for the log stock price as $t \rightarrow \infty$. We now state the first main result, which is a large deviation principle for the re-scaled log stock price $\left(X_{t} / t\right)$ as $t \rightarrow \infty$.

THEOREM 4.1. Consider the process $X$ defined in (3.1) and let $b(y)=$ $\sigma(y)(\alpha y-g(y))-\frac{1}{2} \sigma^{\prime}(y), G(\mu)=\int_{-\infty}^{\infty} b(y) \mu(d y)$. Then under Assumptions 3.1 and 3.2, $X_{t} / t$ satisfies the LDP as $t \rightarrow \infty$ with a good rate function given by

$$
I(x)=\inf _{\mu \in \mathcal{P}(\mathbb{R})}\left[\frac{(x-M(\mu))^{2}}{2 v(\mu)}+I_{Y}(\mu)\right],
$$

where $M(\mu)=-\frac{1}{2} F(\mu)+\rho G(\mu), v(\mu)=\bar{\rho}^{2} F(\mu)$ and $I_{Y}(\mu)$ is the rate function for the occupation measure of $Y$ defined in (2.1).

PROOF. Integrating (3.1), we see that

$$
X_{t}=-\frac{1}{2} \int_{0}^{t} \sigma\left(Y_{s}\right)^{2} d s+\int_{0}^{t} \sigma\left(Y_{s}\right)\left(\rho d W_{s}^{2}+\bar{\rho} d W_{s}^{1}\right) .
$$

If we let $\chi(y)=\int_{y_{0}}^{y} \sigma(u) d u$, then

$$
\begin{aligned}
d \chi\left(Y_{t}\right) & =\sigma\left(Y_{t}\right) d Y_{t}+\frac{1}{2} \sigma^{\prime}\left(Y_{t}\right) d\langle Y\rangle_{t} \\
& =\sigma\left(Y_{t}\right)\left(\left(-\alpha Y_{t}+g\left(Y_{t}\right)\right) d t+d W_{t}^{2}\right)+\frac{1}{2} \sigma^{\prime}\left(Y_{t}\right) d t
\end{aligned}
$$

which we can integrate and re-arrange as follows:

$$
\begin{aligned}
\int_{0}^{t} \sigma\left(Y_{s}\right) d W_{s}^{2} & =\chi\left(Y_{t}\right)+\int_{0}^{t}\left[\sigma\left(Y_{s}\right)\left(\alpha Y_{s}-g\left(Y_{s}\right)\right)-\frac{1}{2} \sigma^{\prime}\left(Y_{s}\right)\right] d s \\
& =\chi\left(Y_{t}\right)+\int_{0}^{t} b\left(Y_{s}\right) d s .
\end{aligned}
$$

Now let $Z_{t}=W_{t}^{1} / t$ and $\hat{X}_{t}=X_{t} / t$. Conditioning on $\left(Y_{s} ; 0 \leq s \leq t\right)$, we obtain

$$
\begin{aligned}
\hat{X}_{t} & \stackrel{d}{=}-\frac{1}{2} F\left(\mu_{t}\right)+\rho\left[G\left(\mu_{t}\right)+\frac{1}{t} \chi\left(Y_{t}\right)\right]+\frac{\bar{\rho}}{t} W_{t F\left(\mu_{t}\right)}^{1} \\
& \stackrel{d}{=}-\frac{1}{2} F\left(\mu_{t}\right)+\rho\left[G\left(\mu_{t}\right)+\frac{1}{t} \chi\left(Y_{t}\right)\right]+\frac{\bar{\rho} \sqrt{F\left(\mu_{t}\right)}}{t} W_{t}^{1} \\
& =M\left(\mu_{t}\right)+\sqrt{v\left(\mu_{t}\right)} Z_{t}+\frac{\rho}{t} \chi\left(Y_{t}\right),
\end{aligned}
$$

where $M(\mu)=-\frac{1}{2} F(\mu)+\rho G(\mu)$ and $v(\mu)=\bar{\rho}^{2} F(\mu)$. From the Gärtner-Ellis theorem, we know that $Z_{t}$ satisfies a large time LDP with good rate function rate 
$\frac{1}{2} z^{2}$, we also know that $\mu_{t}$ satisfies a large time LDP with good rate function $I_{Y}(\mu)$.

Moreover, $Z_{t}$ and $\mu_{t}$ are independent, so we have

$$
\begin{aligned}
\mathcal{I}(z, \mu) & =-\lim _{\delta \rightarrow 0} \lim _{t \rightarrow \infty} \frac{1}{t} \log \mathbb{P}\left(Z_{t} \in B_{\delta}(z), \mu_{t} \in B_{\delta}(\mu)\right) \\
& =-\lim _{\delta \rightarrow 0} \lim _{t \rightarrow \infty} \frac{1}{t}\left[\log \mathbb{P}\left(Z_{t} \in B_{\delta}(z)\right)+\log \mathbb{P}\left(\mu_{t} \in B_{\delta}(\mu)\right)\right] \\
& =\frac{1}{2} z^{2}+I_{Y}(\mu) .
\end{aligned}
$$

Thus, $\left(Z_{t}, \mu_{t}\right)$ satisfies the weak LDP with rate $\mathcal{I}(z, \mu)=\frac{1}{2} z^{2}+I_{Y}(\mu)$. Since $\mu_{t}$ is exponentially tight (by Remark 2.2), for any $c>0$, there exists a compact set $K_{c} \subset \mathcal{P}(\mathbb{R})$ such that $\limsup _{t \rightarrow \infty} \frac{1}{t} \log \mathbb{P}\left(\mu_{t} \notin K_{c}\right) \leq-c$. Thus, for any $c>0$, there exists a compact set $[-\sqrt{2 c}, \sqrt{2 c}] \times K_{c} \subset \mathbb{R} \times \mathcal{P}(\mathbb{R})$ such that

$$
\begin{aligned}
& \limsup _{t \rightarrow \infty} \frac{1}{t} \log \mathbb{P}\left(\left(Z_{t}, \mu_{t}\right) \notin[-\sqrt{2 c}, \sqrt{2 c}] \times K_{c}\right) \\
& \quad \leq \limsup _{t \rightarrow \infty} \frac{1}{t} \log \left[\mathbb{P}\left(\left|Z_{t}\right|>\sqrt{2 c}\right)+\mathbb{P}\left(\mu_{t} \notin K_{c}\right)\right] \\
& \quad \leq \max \left\{\limsup _{t \rightarrow \infty} \frac{1}{t} \log \mathbb{P}\left(\left|Z_{t}\right|>\sqrt{2 c}\right), \limsup _{t \rightarrow \infty} \frac{1}{t} \log \mathbb{P}\left(\mu_{t} \notin K_{c}\right)\right\} \\
& \quad \leq-c .
\end{aligned}
$$

Thus, $\left(Z_{t}, \mu_{t}\right)$ is exponentially tight, so $\left(Z_{t}, \mu_{t}\right)$ satisfies the full LDP and (by Lemma 1.2.18b in [3]) the rate function $\mathcal{I}(z, \mu)$ is good. From (4.2), we have

$$
\hat{X}_{t} \stackrel{d}{=} \tilde{X}_{t}:=\varphi\left(Z_{t}, \mu_{t}\right)+\frac{\rho}{t} \chi\left(Y_{t}\right),
$$

where $\varphi: \mathbb{R} \times \mathcal{P}(\mathbb{R}) \mapsto \mathbb{R}$ is given by $\varphi(z, \mu)=M(\mu)+\sqrt{v(\mu)} z$. Similarly, define

$$
\tilde{X}_{t}^{m}=\varphi^{m}\left(Z_{t}, \mu_{t}\right),
$$

where $\varphi^{m}(z)=M^{m}(\mu)+\sqrt{v^{m}(\mu)} z$, where we have truncated the integrands in $M(\mu)$ and $\nu(\mu)$ to get

$$
\begin{aligned}
M^{m}(\mu)= & \int\left[\left(-\frac{1}{2} \sigma^{2}(y)+\rho b(y)\right) 1_{\{|y| \leq m\}}+\left(-\frac{1}{2} \sigma^{2}(m)+\rho b(m)\right) 1_{\{y>m\}}\right. \\
& \left.+\left(-\frac{1}{2} \sigma^{2}(-m)+\rho b(-m)\right) 1_{\{y<-m\}}\right] \mu(d y)
\end{aligned}
$$

and

$$
v^{m}(\mu)=\bar{\rho}^{2} \int\left[\sigma^{2}(y) 1_{|y| \leq m}+\sigma^{2}(m) 1_{y>m}+\sigma^{2}(-m) 1_{y<-m}\right] \mu(d y) .
$$


Since the integrands are bounded and continuous functions of $\mathbb{R}, M^{m}(\mu)$ and $v^{m}(\mu)$ are continuous functionals of $\mu$ under the weak topology. Using the Hölder continuity of the square root function: $|\sqrt{x}-\sqrt{y}| \leq \sqrt{|x-y|}$, we have

$$
\left|\tilde{X}_{t}-\tilde{X}_{t}^{m}\right| \leq\left|M\left(\mu_{t}\right)-M^{m}\left(\mu_{t}\right)\right|+\sqrt{\left|v\left(\mu_{t}\right)-v^{m}\left(\mu_{t}\right)\right|}\left|Z_{t}\right|+\left|\frac{\rho}{t} \chi\left(Y_{t}\right)\right| .
$$

Then

$$
\begin{aligned}
& \mathbb{P}\left(\left|\tilde{X}_{t}-\tilde{X}_{t}^{m}\right|>\delta\right) \\
& \leq \mathbb{P}\left(\left|M\left(\mu_{t}\right)-M^{m}\left(\mu_{t}\right)\right|+\sqrt{\left|v\left(\mu_{t}\right)-v^{m}\left(\mu_{t}\right)\right|}\left|Z_{t}\right|+\left|\frac{\rho}{t} \chi\left(Y_{t}\right)\right|>\delta\right) \\
& \leq \mathbb{P}\left(\left|M\left(\mu_{t}\right)-M^{m}\left(\mu_{t}\right)\right|+\sqrt{\left|v\left(\mu_{t}\right)-v^{m}\left(\mu_{t}\right)\right|}\left|Z_{t}\right|>\frac{1}{2} \delta\right) \\
& +\mathbb{P}\left(\left|\frac{\rho}{t} \chi\left(Y_{t}\right)\right|>\frac{1}{2} \delta\right) \\
& \leq \mathbb{E}\left(1_{\left|M\left(\mu_{t}\right)-M^{m}\left(\mu_{t}\right)\right|+\sqrt{\left|v\left(\mu_{t}\right)-v^{m}\left(\mu_{t}\right)\right| \mid} Z_{t} \mid>\frac{1}{2} \delta} 1_{I_{Y}\left(\mu_{t}\right) \leq c}\right)+\mathbb{P}\left(I_{Y}\left(\mu_{t}\right)>c\right) \\
& +\mathbb{P}\left(\left|\frac{\rho}{t} \chi\left(Y_{t}\right)\right|>\frac{1}{2} \delta\right) .
\end{aligned}
$$

We will use the following lemma in the subsequent proof.

LEMMA 4.1. Consider the $Y$ process defined in (3.1). Then for $v>0$ we have $\limsup _{t \rightarrow \infty} \frac{1}{t} \log \mathbb{P}\left(\left|\frac{1}{t} \chi\left(Y_{t}\right)\right|>v\right)=-\infty$, where $\chi(\cdot)$ is defined as in the proof of Theorem 4.1 .

\section{Proof. See Appendix C.}

Recall that $M(\mu)=-\frac{1}{2} F(\mu)+\rho G(\mu)$ and $\nu(\mu)=\bar{\rho}^{2} F(\mu)$. Then if $I_{Y}(\mu) \leq c$, from Lemmas A.1 to A.4 in Appendix A we obtain

$$
\left|M(\mu)-M^{m}(\mu)\right| \vee\left|v(\mu)-v^{m}(\mu)\right| \leq \gamma_{m}(c),
$$

where $\gamma_{m}(c)=2 A\left(\frac{1}{m^{2}}+\frac{1}{m^{2-q}}\right)\left(K_{2}(\alpha) c+K_{3}(\alpha)\right)$ for some $A>0$ and $q=1+p$, and $\gamma_{m}(c) \rightarrow 0$ as $m \rightarrow \infty$. Now let $\zeta=\mathbb{P}\left(I_{Y}\left(\mu_{t}\right)>c\right)+\mathbb{P}\left(\left|\frac{\rho}{t} \chi\left(Y_{t}\right)\right|>\frac{1}{2} \delta\right)$. Then using (4.5), we can now further bound the right-hand side of (4.4) as follows:

$$
\begin{aligned}
\mathbb{P}\left(\left|\tilde{X}_{t}-\tilde{X}_{t}^{m}\right|>\delta\right) & \leq \mathbb{E}\left(1_{\gamma_{m}+\sqrt{\gamma_{m}}\left|Z_{t}\right|>\frac{1}{2} \delta} 1_{I_{Y}\left(\mu_{t}\right) \leq c}\right)+\zeta \\
& \leq \mathbb{P}\left(\gamma_{m}(c)+\sqrt{\gamma_{m}(c)}\left|Z_{t}\right|>\frac{1}{2} \delta\right)+\zeta \\
& \leq \mathbb{P}\left(\left|Z_{t}\right|>\frac{\frac{1}{2} \delta-\gamma_{m}(c)}{\sqrt{\gamma_{m}(c)}}\right)+\zeta
\end{aligned}
$$


Letting $t \rightarrow \infty$ and using the LDP for $\mu_{t}$ and the LDP for $Z_{t}$ and Lemma 4.1 we obtain

$$
\limsup _{t \rightarrow \infty} \frac{1}{t} \log \mathbb{P}\left(\left|\tilde{X}_{t}-\tilde{X}_{t}^{m}\right|>\delta\right) \leq-\frac{\left(\frac{1}{2} \delta-\gamma_{m}(c)\right)^{2}}{2 \gamma_{m}(c)} \wedge c .
$$

Now set $c=c(m)=m^{\beta}$ where $\beta \in(0,2-q)$. Then $\gamma_{m}^{*}=\gamma_{m}(c(m))=2 A\left(\frac{1}{m^{2}}+\right.$ $\left.\frac{1}{m^{2-q}}\right)\left(K_{2}(\alpha) m^{\beta}+K_{3}(\alpha)\right) \rightarrow 0$ as $m \rightarrow \infty$ and

$$
\lim _{m \rightarrow \infty} \limsup _{t \rightarrow \infty} \frac{1}{t} \log \mathbb{P}\left(\left|\tilde{X}_{t}-\tilde{X}_{t}^{m}\right|>\delta\right) \leq-\infty .
$$

Thus, $X_{t}^{m}$ is an exponentially good approximation to $X_{t}$ in the sense of Definition 4.2.14 in [3]. From the analysis above, we have

$$
\begin{aligned}
& \limsup _{m \rightarrow \infty} \sup _{(z, \mu): \mathcal{I}(z, \mu) \leq R}\left|\varphi(z, \mu)-\varphi^{m}(z, \mu)\right| \\
& \quad \leq \limsup _{m \rightarrow \infty} \sup _{(z, \mu): \frac{1}{2} z^{2}+I_{Y}(\mu) \leq R}|| M(\mu)-M^{m}(\mu)\left|+\sqrt{v(\mu)-v^{m}(\mu)}\right| z|| \\
& \quad \leq \limsup _{m \rightarrow \infty} \sup _{(z, \mu): \frac{1}{2} z^{2}+I_{Y}(\mu) \leq R}\left|\gamma_{m}(R)+\sqrt{\gamma_{m}}(R) \sqrt{2 R}\right| \\
& \quad=0 .
\end{aligned}
$$

Thus, by Theorem 4.2.23 in [3], $\hat{X}_{t}$ satisfies the LDP with good rate function

$$
I(x)=\inf _{(z, \mu): M(\mu)+\sqrt{\nu(\mu)} z=x}\left[\frac{1}{2} z^{2}+I_{Y}(\mu)\right] .
$$

But $v(\mu)=\bar{\rho}^{2} \int_{-\infty}^{\infty} \sigma^{2}(y) \mu(d y)>0$ because $\sigma^{2}$ is strictly positive. Thus, we can re-write the right-hand side of (4.6) as $\inf _{\mu \in \mathcal{P}(\mathbb{R})}\left[\frac{(x-M(\mu))^{2}}{2 v(\mu)}+I_{Y}(\mu)\right]$.

4.2. Properties of the rate function $I(x)$. The following two corollaries establish some basic properties of $I(x)$.

COROLLARY 4.2. The infimum of $I(x)$ in (4.1) is attained uniquely at

$$
x_{\min }=M\left(\mu_{\infty}\right)=-\frac{1}{2} \bar{\sigma}^{2},
$$

where $M(\cdot)$ is defined as in Theorem 4.1 and $\bar{\sigma}$ is defined in (3.5).

Proof. Let $I(x, \mu)=\frac{(x-M(\mu))^{2}}{2 v(\mu)}+I_{Y}(\mu)$. Then, by $(4.1), \quad I(x)=$ $\inf _{\mu \in \mathcal{P}(\mathbb{R})} I(x, \mu)$. Setting $\mu=\mu_{\infty}$ we have $I\left(x_{\min }, \mu_{\infty}\right)=\frac{\left(x_{\min }-M\left(\mu_{\infty}\right)\right)^{2}}{2 v\left(\mu_{\infty}\right)^{2}}+$ $I_{Y}\left(\mu_{\infty}\right)=0$. Therefore,

$$
0 \leq I\left(x_{\min }\right)=\inf _{\mu \in \mathcal{P}(\mathbb{R})} I\left(x_{\min }, \mu\right) \leq I\left(x_{\min }, \mu_{\infty}\right)=0
$$

so $I\left(x_{\min }\right)=0$. 
We show that $x_{\min }$ is the unique minimum by contradiction. Suppose there exists an $x \neq x_{\text {min }}$ such that $\lim _{n \rightarrow \infty} I\left(x, \mu_{n}\right)=0$ for some sequence $\left(\mu_{n}\right)$ with $\mu_{n} \in$ $\mathcal{P}(\mathbb{R})$. If $I\left(x, \mu_{n}\right) \rightarrow 0$ as $n \rightarrow \infty$ then $I_{Y}\left(\mu_{n}\right) \rightarrow 0$ and $M\left(\mu_{n}\right) \rightarrow x$ as $n \rightarrow \infty$. We first show that $\left(\mu_{n}\right)$ is a tight sequence. For any $k>0$,

$$
k^{2} \mu_{n}[-k, k]^{c} \leq \int_{[-k, k]^{c}} y^{2} \mu_{n}(d y) \leq K_{2}(\alpha) I_{Y}\left(\mu_{n}\right)+K_{3}(\alpha),
$$

where we have used Lemma 2.2 for the last inequality. Since $I_{Y}\left(\mu_{n}\right) \rightarrow 0$ as $n \rightarrow$ $\infty$, we can find a $C<\infty$ such that $\sup _{n} I_{Y}\left(\mu_{n}\right) \leq C$. Hence, $k^{2} \mu_{n}[-k, k]^{c} \leq$ $K_{2}(\alpha) C+K_{3}(\alpha)$ for all $n$. Thus, given $\varepsilon>0$, we can choose $k$ large enough such that

$$
\sup _{n} \mu_{n}[-k, k]^{c} \leq \frac{K_{2}(\alpha) C+K_{3}(\alpha)}{k^{2}}<\varepsilon,
$$

so $\left(\mu_{n}\right)$ is tight as required.

Hence, $\left(\mu_{n}\right)$ has a convergent subsequence. Without loss of generality, we denote the convergent subsequence by $\left(\mu_{n}\right)$ and let $\mu$ denote the limit point. Then $I_{Y}(\mu)=0$ by lower semicontinuity of $I_{Y}$ [i.e., $I_{Y}(\mu) \leq \liminf _{\mu_{n} \rightarrow \mu} I_{Y}\left(\mu_{n}\right)=0$ ] and by uniqueness of minimizer of $I_{Y}$ we obtain $\mu=\mu_{\infty}$. We will next show that $M\left(\mu_{n}\right) \rightarrow M\left(\mu_{\infty}\right)=x_{\min }$ which gives the contradiction.

Let $m>0$. Then

$$
\begin{aligned}
\left|M\left(\mu_{n}\right)-M\left(\mu_{\infty}\right)\right| \leq & \left|M_{m}\left(\mu_{n}\right)-M_{m}\left(\mu_{\infty}\right)\right|+\left|M\left(\mu_{n}\right)-M_{m}\left(\mu_{n}\right)\right| \\
& +\left|M\left(\mu_{\infty}\right)-M_{m}\left(\mu_{\infty}\right)\right| \\
\leq & \left|M_{m}\left(\mu_{n}\right)-M_{m}\left(\mu_{\infty}\right)\right|+c(m)\left(C_{1} I_{Y}\left(\mu_{n}\right)+C_{2}\right) \\
& +c(m)\left(C_{1} I_{Y}\left(\mu_{\infty}\right)+C_{2}\right)
\end{aligned}
$$

[where we have applied Lemma A.1, and $C_{1}, C_{2}$ are constants and $c(m)=1 / m^{2}+$ $1 / m^{2-q}$ for some $\left.q \in(0,2)\right]$

$$
=\left|M_{m}\left(\mu_{n}\right)-M_{m}\left(\mu_{\infty}\right)\right|+c(m)\left(C_{1} I_{Y}\left(\mu_{n}\right)+C_{2}\right)+c(m) C_{2} .
$$

Taking $n \rightarrow \infty$, we see that

$$
\lim _{n \rightarrow \infty}\left|M\left(\mu_{n}\right)-M\left(\mu_{\infty}\right)\right| \leq 0+2 c(m) C_{2}
$$

because $M_{m}$ is a continuous functional. Since this holds for any arbitrary $m>0$, taking $m \rightarrow \infty$ and noting that $c(m) \rightarrow 0$ as $m \rightarrow \infty$, we get $M\left(\mu_{n}\right) \rightarrow M\left(\mu_{\infty}\right)=$ $x_{\min }$.

Finally, using the definition of $M(\cdot)$ in Theorem 4.1, we find that $M\left(\mu_{\infty}\right)=$ $-\frac{1}{2} \bar{\sigma}^{2}+\rho \bar{b}$ where $\bar{b}=\int_{-\infty}^{\infty} b(y) \mu_{\infty}(y) d y$. Recall that $b(y)$ is defined in Theo- 
rem 4.1 as $b(y)=(\alpha y-g(y)) \sigma(y)-\frac{1}{2} \sigma^{\prime}(y)$. Then we have

$$
\begin{aligned}
\int b(y) \mu_{\infty}(d y) & \\
= & \text { const. } \times\left[\int_{-\infty}^{\infty}[\sigma(y)(\alpha y-g(y))] e^{2 \int_{y_{0}}^{y} g(u) d u} e^{-\alpha y^{2}} d y\right. \\
& \left.-\int_{-\infty}^{\infty} \frac{1}{2} \sigma^{\prime}(y) e^{-\alpha y^{2}} e^{2 \int_{y_{0}}^{y} g(u) d u} d y\right] \\
= & \text { const. } \times\left[\int_{-\infty}^{\infty}[\sigma(y)(\alpha y-g(y))] e^{2 \int_{y_{0}}^{y} g(u) d u} e^{-\alpha y^{2}} d y\right. \\
& \left.+\int_{-\infty}^{\infty} \frac{1}{2} \sigma(y) e^{-\alpha y^{2}} e^{2 \int_{y_{0}}^{y} g(u) d u}(-2 \alpha y+2 g(y)) d y\right] \\
= & 0,
\end{aligned}
$$

where we have integrated by parts in the second expression of the last line. Thus, we see that $x_{\min }=-\frac{1}{2} \bar{\sigma}^{2}$.

COROLlary 4.3. I $\quad$ (x) in (4.1) is continuous.

ProOf. Let $I(x, \mu)$ be as defined in Corollary 4.2. Then $I(x, \mu)$ is upper semicontinuous in $x$ for $\mu$ fixed, and $I(x)=\inf _{\mu} I(x, \mu)$. The pointwise supremum of a family of LSC functions is LSC (see, e.g., Lemma 2.41 on page 43 in [1]), hence the pointwise infimum of a family of USC functions is USC, so $I(x)$ is USC. But $I(x)$ is also a rate function, hence $I$ is also LSC.

\subsection{The case $x=0$ with $\rho=0$ - the Rayleigh-Ritz formula.}

Corollary 4.4. For $x=0, \rho=0, I(0)$ reduces to

$$
\begin{aligned}
I(0)=\lambda_{1} & =\inf _{\mu \in \mathcal{P}(\mathbb{R})}\left[\frac{1}{8} F(\mu)+I_{Y}(\mu)\right] \\
& =\inf _{\psi \in L^{2}\left(\mu_{\infty}\right):\|\psi\|_{2}=1} \int_{-\infty}^{\infty}\left[\frac{1}{8} \sigma^{2}(y) \psi(y)^{2}+\frac{1}{2} \psi^{\prime}(y)^{2}\right] \mu_{\infty}(y) d y .
\end{aligned}
$$

PROOF. The first equality in (4.9) just follows by setting $x=0$ in (4.1) and simplifying. The second equality just follows by re-writing $\mu$ in terms of $\psi$.

REMARK 4.5. (4.9) is the classical Rayleigh-Ritz formula for the lowest eigenvalue $\lambda_{1}$ for the Sturm-Liouville problem $(-\alpha y+g(y)) u^{\prime}+\frac{1}{2} u^{\prime \prime}-$ $\frac{1}{8} \sigma^{2}(y) u=-\lambda_{1} u$ (see page 2 in [4] for more details). 
4.4. A general vol-of-vol coefficient. For a more general model of the form,

$$
\left\{\begin{array}{l}
d X_{t}=-\frac{1}{2} \sigma\left(V_{t}\right)^{2} d t+\sigma\left(V_{t}\right)\left(\rho d W_{t}^{2}+\bar{\rho} d W_{t}^{1}\right) \\
d V_{t}=\left(-\alpha V_{t}+g\left(V_{t}\right)\right) d t+\beta\left(V_{t}\right) d W_{t}^{2}
\end{array}\right.
$$

for $g, \sigma$ satisfying the same conditions as before, $\beta \in C^{4}$ with bounded first derivative (so $\beta$ is Lipschitz), $0<\beta \leq \beta(v) \leq \bar{\beta}<\infty, \beta(v) \rightarrow \beta_{\infty}$ as $|v| \rightarrow \infty$ and $\frac{1}{\beta(v)}-\frac{1}{\beta_{\infty}}=O\left(1 / 1+|v|^{\gamma}\right)$ for some $\gamma>0$, then making the transformation $Y_{t}=U\left(V_{t}\right)$, where $U(v)=\int_{0}^{v} \frac{d z}{\beta(z)}$, we find that

$$
\begin{aligned}
d Y_{t}= & U^{\prime}\left(V_{t}\right) d V_{t}+\frac{1}{2} U^{\prime \prime}\left(V_{t}\right) d\langle V\rangle_{t} \\
= & U^{\prime}\left(V_{t}\right)\left[\left(-\alpha V_{t}+g\left(V_{t}\right)\right) d t+\beta\left(V_{t}\right) d W_{t}^{2}\right]+\frac{1}{2} U^{\prime \prime}\left(V_{t}\right) \beta\left(V_{t}\right)^{2} d t \\
= & \frac{1}{\beta\left(V_{t}\right)}\left[\left(-\alpha V_{t}+g\left(V_{t}\right)\right) d t\right]+d W_{t}^{2}-\frac{1}{2} \beta^{\prime}\left(V_{t}\right) d t \\
= & {\left[-\frac{\alpha}{\beta\left(V_{t}\right)} V_{t}+\frac{g\left(V_{t}\right)}{\beta\left(V_{t}\right)}-\frac{1}{2} \beta^{\prime}\left(V_{t}\right)\right] d t+d W_{t}^{2} } \\
= & {\left[-\alpha Y_{t}+\left(\alpha Y_{t}-\frac{\alpha}{\beta\left(V_{t}\right)} V_{t}\right)+\left(\frac{g\left(V_{t}\right)}{\beta\left(V_{t}\right)}-\frac{1}{2} \beta^{\prime}\left(V_{t}\right)\right)\right] d t+d W_{t}^{2} } \\
= & {\left[-\alpha Y_{t}+\left(\alpha Y_{t}-\frac{\alpha}{\beta\left(U^{-1}\left(Y_{t}\right)\right)} U^{-1}\left(Y_{t}\right)\right)\right.} \\
& \left.+\left(\frac{g\left(U^{-1}\left(Y_{t}\right)\right)}{\beta\left(U^{-1}\left(Y_{t}\right)\right)}-\frac{1}{2} \beta^{\prime}\left(U^{-1}\left(Y_{t}\right)\right)\right)\right] d t+d W_{t}^{2} .
\end{aligned}
$$

We need to show that the terms $\alpha Y_{t}-\frac{\alpha}{\beta\left(U^{-1}\left(Y_{t}\right)\right)} U^{-1}\left(Y_{t}\right)$ and $\frac{g\left(U^{-1}\left(Y_{t}\right)\right)}{\beta\left(U^{-1}\left(Y_{t}\right)\right)}-$ $\frac{1}{2} \beta^{\prime}\left(U^{-1}\left(Y_{t}\right)\right)$ satisfy the sublinear growth condition in Assumption 3.1. Henceforth, "sublinear growth" will mean that equation (3.2) is satisfied.

We first look at the term $\frac{g\left(U^{-1}\left(Y_{t}\right)\right)}{\beta\left(U^{-1}\left(Y_{t}\right)\right)}-\frac{1}{2} \beta^{\prime}\left(U^{-1}\left(Y_{t}\right)\right)$. Since $1 / \beta(\cdot)$ and $\beta^{\prime}(\cdot)$ are bounded functions, it is sufficient to show that $g\left(U^{-1}\left(Y_{t}\right)\right)$ has sublinear growth in $Y_{t}$. By the definition of $Y$ and bounds on $\beta(\cdot)$, we get $V_{t} / \bar{\beta} \leq Y_{t} \leq V_{t} / \underline{\beta}$ which then gives us the inequality $\beta Y_{t} \leq V_{t}=U^{-1}\left(Y_{t}\right) \leq \bar{\beta} Y_{t}$. Since $g$ has sublinear growth and $V_{t}$ grows linearly with $Y_{t}$, we get that $g\left(U^{-1}\left(Y_{t}\right)\right)$ is a sub linear function of $Y_{t}$.

We next show that $\left|y-\frac{U^{-1}(y)}{\beta\left(U^{-1}(y)\right)}\right| \leq$ constant $\times(1+|y|)^{\delta}$ for some $\delta \in(0,1)$. By definition of $Y$ and properties of $\beta(\cdot)$, we get

$$
y=U(v)=\int_{0}^{v} \frac{1}{\beta(z)} d z=\frac{v}{\beta_{\infty}}+\int_{0}^{v}\left(\frac{1}{\beta(z)}-\frac{1}{\beta_{\infty}}\right) d z=\frac{v}{\beta_{\infty}}+O\left(1+|v|^{1-\gamma}\right)
$$


and

$$
\frac{v}{\beta(v)}=\frac{v}{\beta_{\infty}}+O\left(1+|v|^{1-\gamma}\right) .
$$

Putting this together, we get

$$
y-\frac{U^{-1}(y)}{\beta\left(U^{-1}(y)\right)}=U(v)-\frac{v}{\beta(v)}=O\left(1+|v|^{1-\gamma}\right) .
$$

Since $V_{t}$ grows linearly with $Y_{t}$, we get $\left|y-\frac{U^{-1}(y)}{\beta\left(U^{-1}(y)\right)}\right|=O\left(1+|y|^{1-\gamma}\right)$. So $\mid y-$ $\frac{U^{-1}(y)}{\beta\left(U^{-1}(y)\right)} \mid \leq$ constant if $\gamma>1$ and $\left|y-\frac{U^{-1}(y)}{\beta\left(U^{-1}(y)\right)}\right| \leq$ constant $\times\left(1+|y|^{1-\gamma}\right)$ if $\gamma \in(0,1)$. Thus,

$$
\left\{\begin{array}{l}
d X_{t}=-\frac{1}{2} \tilde{\sigma}\left(Y_{t}\right)^{2} d t+\tilde{\sigma}\left(Y_{t}\right)\left(\rho d W_{t}^{2}+\bar{\rho} d W_{t}^{1}\right), \\
d Y_{t}=\left(-\alpha Y_{t}+\tilde{g}\left(Y_{t}\right)\right) d t+d W_{t}^{2}
\end{array}\right.
$$

for some $\tilde{\sigma}, \tilde{g}$ which satisfy Assumptions 3.1 and 3.2, so we are back to a model of the form in (3.1), and thus the main result in Theorem 4.1 still holds. If we want to impose less stringent conditions on $\beta$, we would have to manually verify the upper bound conditions 1-5 and the lower bound conditions A, B in Section 2.1.

5. Call options and implied volatility. We now verify the martingale property for $S_{t}=e^{X_{t}}$. This will be used to define the Share measure $\mathbb{P}^{*}$ below.

Proposition 5.1. $\left(S_{t}\right)_{0 \leq t<\infty}$ defined in (3.1) is a martingale.

Proof. See Appendix D.

We consider the family of probability measures $\mathbb{P}_{T}^{S}(A):=\frac{1}{S_{0}} \mathbb{E}\left(S_{T} 1_{A}\right)$ defined for each $T>0$, for $A \in \mathcal{F}_{t}$ and $t \leq T\left[\mathbb{P}_{T}^{S}\right.$ is a probability measure on $\mathcal{F}_{T}$ because $\left(S_{t}\right)_{0 \leq t \leq T}$ is a martingale by Proposition 5.1]. From Girsanov's theorem, we have that

$$
\left\{\begin{array}{l}
d X_{t}=\frac{1}{2} \sigma\left(Y_{t}\right)^{2} d t+\sigma\left(Y_{t}\right)\left(\rho d W_{t}^{* 2}+\bar{\rho} d W_{t}^{* 1}\right), \\
d Y_{t}=\left(-\alpha Y_{t}+g\left(Y_{t}\right)+\rho \sigma\left(Y_{t}\right)\right) d t+d W_{t}^{* 2},
\end{array}\right.
$$

where $W_{t}^{* 1}, W_{t}^{* 2}$ are independent $\mathbb{P}^{S}$-Brownian motions. Let $\mathbb{P}^{*}$ be a probability measure under which $(X, Y)$ satisfies (5.1) for all $t>0$ with $X_{0}=0$ and $Y_{0}=y_{0}$.

Proposition 5.2. $X_{t} / t$ satisfies the LDP under $\mathbb{P}^{*}$ as $t \rightarrow \infty$ with a good rate function given by

$$
I^{*}(x)=\inf _{\mu \in \mathcal{P}(\mathbb{R})}\left[\frac{\left(x-M^{*}(\mu)\right)^{2}}{2 v(\mu)}+I_{Y}(\mu)\right]
$$


where $M^{*}(\mu)=\frac{1}{2} F(\mu)+\rho G^{*}(\mu)$, where $G^{*}(\mu)=\int_{-\infty}^{\infty}[(\alpha y-g(y)-$ $\left.\rho \sigma(y)) \sigma(y)-\frac{1}{2} \sigma^{\prime}(y)\right] \mu(d y)$, and the minimum of $I^{*}(x)$ is attained uniquely at $x_{\min }^{*}=M^{*}\left(\mu_{\infty}^{*}\right)$; where $\mu_{\infty}^{*}$ is the invariant distribution of the $Y$ process under $\mathbb{P}^{*}$.

PROOF. If we let $\tilde{g}(y)=g(y)+\rho \sigma(y)$, then $\tilde{g}$ also has sublinear growth, and the proof then just follows by an almost identical argument to the proofs of Theorem 4.1 and Corollary 4.2.

COROLlary 5.3. The unique minimizers $x_{\min }$ and $x_{\min }^{*}$ of the rate functions $I$ and $I^{*}$, respectively (defined in Corollary 4.2 and Proposition 5.2, resp.), satisfy the inequality $x_{\min }^{*}>x_{\min }$.

PROOF. Recall the formula of the invariant density for the perturbed OU process given in (2.5). Then $\mu_{\infty}^{*}(y)=\frac{e^{-\alpha y^{2}} e^{2 \int_{y_{0}}^{y} \tilde{g}(u) d u}}{\int_{-\infty}^{\infty} e^{-\alpha u^{2}} e^{2 \int_{y_{0}}^{u} \tilde{g}(v) d v d u} d u}$, where $\tilde{g}(y)=g(y)+$ $\rho \sigma(y)$ and $\mu_{\infty}(y)=\frac{e^{-\alpha y^{2}} e^{2 \int_{y}^{y} g(u) d u}}{\int_{-\infty}^{\infty} e^{-\alpha u^{2}} e^{2 \int_{y_{0}}^{u} g(v) d v d u} d u}$. Observe that

$$
\begin{aligned}
G^{*}\left(\mu_{\infty}^{*}\right) & =\text { const } \cdot \int_{-\infty}^{\infty}\left[(\alpha y-\tilde{g}(y)) \sigma(y)-\frac{1}{2} \sigma^{\prime}(y)\right] e^{-\alpha y^{2}} e^{2 \int_{y_{0}}^{y} \tilde{g}(u) d u} d y \\
& =\text { const } \cdot \int_{-\infty}^{\infty}-\frac{1}{2}\left[\frac{d}{d y}\left(\sigma(y) e^{-\alpha y^{2}} e^{2 \int_{y_{0}}^{y} \tilde{g}(u) d u}\right)\right] d y=0 .
\end{aligned}
$$

Similarly, $G\left(\mu_{\infty}\right)=0$. Thus,

$$
\begin{aligned}
x_{\min } & =-\frac{1}{2} F\left(\mu_{\infty}\right)+\rho G\left(\mu_{\infty}\right)=-\frac{1}{2} F\left(\mu_{\infty}\right)<F\left(\mu_{\infty}^{*}\right) \\
& =\frac{1}{2} F\left(\mu_{\infty}^{*}\right)+\rho G^{*}\left(\mu_{\infty}^{*}\right)=x_{\min }^{*} .
\end{aligned}
$$

By Proposition 5.2, that is, the LDP for $\left(X_{t} / t\right)$ under $\mathbb{P}^{*}$, and the continuity of the rate function $I^{*}$, we obtain the following corollary, which will be used to characterize the large-time behaviour of call option prices.

COROLLARY 5.4. For the model in (3.1), we have the following large-time behaviour for digital call options:

$$
\begin{aligned}
& \lim _{t \rightarrow \infty} \frac{1}{t} \log \mathbb{P}^{*}\left(X_{t}>x t\right)=-\Lambda^{*}(x) \quad\left(x>x_{\min }^{*}\right), \\
& \lim _{t \rightarrow \infty} \frac{1}{t} \log \mathbb{P}^{*}\left(X_{t}<x t\right)=-\Lambda^{*}(x) \quad\left(x<x_{\min }^{*}\right),
\end{aligned}
$$

where

$$
\Lambda^{*}(x)= \begin{cases}\inf _{y>x} I^{*}(y), & \text { if } x \geq x_{\min }^{*}, \\ \inf _{y<x} I^{*}(y), & \text { if } x \leq x_{\min }^{*}\end{cases}
$$


REMARK 5.5. From the definition of $\Lambda^{*}$, we see that $\Lambda^{*}$ is nonincreasing for $x<x_{\min }^{*}$ and nondecreasing for $x>x_{\min }^{*}$, and [by the continuity of $I^{*}(x)$, which can be proved by a similar argument to Corollary 4.3] $\Lambda^{*}$ is continuous.

Recall that the payoff of a European call option of strike $K$ is $\mathbb{E}\left(S_{t}-K\right)^{+}$, and the payoff of a European put option with strike $K$ is $\mathbb{E}\left(K-S_{t}\right)^{+}$.

COROLlary 5.6. For the model in (3.1), we have the following large-time asymptotic behaviour for put/call options in the large-time, large log-moneyness regime:

$$
\begin{aligned}
-\lim _{t \rightarrow \infty} \frac{1}{t} \log \mathbb{E}\left(S_{t}-S_{0} e^{x t}\right)^{+} & =\Lambda^{*}(x) & & \left(x \geq x_{\min }^{*}\right), \\
(5.2)-\lim _{t \rightarrow \infty} \frac{1}{t} \log \left[S_{0}-\mathbb{E}\left(S_{t}-S_{0} e^{x t}\right)^{+}\right] & =\Lambda^{*}(x) & & \left(x_{\min } \leq x \leq x_{\min }^{*}\right), \\
-\lim _{t \rightarrow \infty} \frac{1}{t} \log \left(\mathbb{E}\left(S_{0} e^{x t}-S_{t}\right)^{+}\right) & =\Lambda^{*}(x) & & \left(x \leq x_{\min }\right),
\end{aligned}
$$

PROOF. This is now a standard result; see, for example, Corollary 2.4 in [13].

5.1. Implied volatility. Using the same proofs as in Corollary 1.7 and Corollary 2.17 in [13] for the Heston model (see also Theorem 14 in [18] for a general affine model), we have the following asymptotic behaviour in the large-time, large $\log$-moneyness regime, where $\hat{\sigma}_{t}(x t)$ is the implied volatility of a put/call option with strike $S_{0} e^{x t}$ for model in (3.1):

$$
\begin{aligned}
\hat{\sigma}_{\infty}(x)^{2} & =\lim _{t \rightarrow \infty} \hat{\sigma}_{t}^{2}(x t) \\
& = \begin{cases}2\left(2 \Lambda^{*}(x)+x-2 \sqrt{\left.\Lambda^{*}(x)^{2}+\Lambda^{*}(x) x\right)}\right. & \left(x \notin\left(x_{\min }, x_{\min }^{*}\right)\right), \\
2\left(2 \Lambda^{*}(x)+x+2 \sqrt{\left.\Lambda^{*}(x)^{2}+\Lambda^{*}(x) x\right)}\right. & \left(x \in\left(x_{\min }, x_{\min }^{*}\right)\right)\end{cases}
\end{aligned}
$$

and we see that $\hat{\sigma}_{\infty}(0)^{2}=8 \Lambda^{*}(0)$. We omit the details for the sake of brevity.

6. Numerical implementation and results. Recall that the rate function $I(x)$ for $X_{t} / t$ under the model in (3.1) is given by $I(x)=\inf _{\mu \in \mathcal{P}(\mathbb{R})}\left[\frac{(x-M(\mu))^{2}}{2 v(\mu)}+\right.$ $I_{Y}(\mu)$ ]. If $g(y) \equiv 0$, then using the simpler representation for the rate function in (2.3), we can re-write $I(x)$ as

$$
I(x)=\inf _{\psi \in L^{2}\left(\mu_{\infty}\right):\|\psi\|_{2}=1}\left[\frac{(x-M)^{2}}{2 v}+\frac{1}{2} \int_{-\infty}^{\infty} \psi^{\prime}(y)^{2} \mu_{\infty}(y) d y\right]
$$

where now $M=M(\psi)=\int_{-\infty}^{\infty} m(y) \psi(y)^{2} \mu_{\infty}(y) d y, m(y)=-\frac{1}{2} \sigma^{2}(y)+\rho b(y)$ and $v=v(\psi)=\bar{\rho}^{2} \int_{-\infty}^{\infty} \sigma(y)^{2} \psi(y)^{2} \mu_{\infty}(y) d y$ [the constraint under the inf is just shorthand for $\left.\int_{-\infty}^{\infty} \psi(y)^{2} \mu_{\infty}(y) d y=1\right]$. 


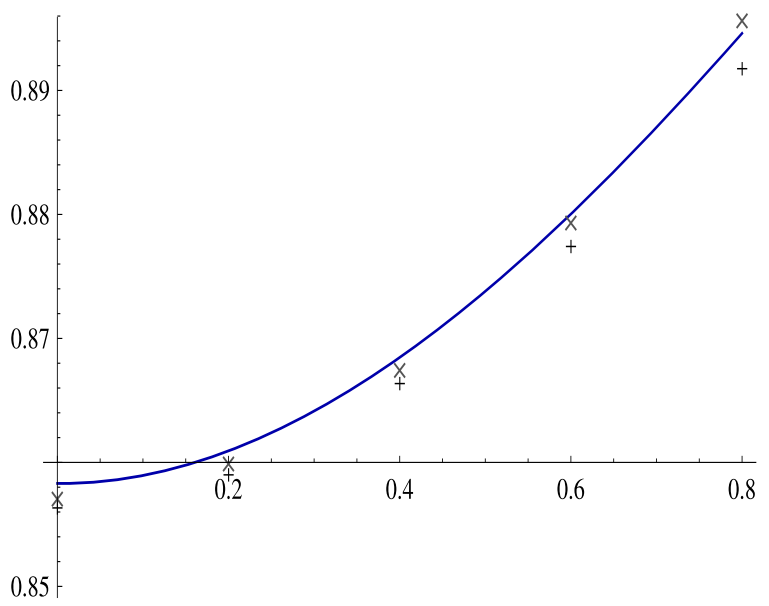

FIG. 1. Here, we have plotted the right half of the (symmetric) asymptotic implied volatility $\hat{\sigma}_{\infty}(x)$ for the Ornstein-Uhlenbeck model with $\rho=0, \alpha=1$ and $\sigma(y)=\sqrt{\log \left(1+e^{y}\right)}$ (solid blue line) using the Ritz method with the NMinimize command in Mathematica and $n=7$, and the values obtained using Monte Carlo simulation for $t=75$ years (grey diagonal crosses) and $t=30$ years (black crosses). For the Monte Carlo, we use 1,000,000 simulations and 1000 time steps and we use the usual conditioning trick for $\rho=0$ by simulating the integrated variance $\int_{0}^{t} \sigma\left(Y_{S}\right)^{2} d s$ and then plugging this into the Black-Scholes formula. In this case, $x_{\min }^{*}=0.376131$ and $x_{\min }=-x_{\min }^{*}$. Note that $\sigma(y) \sim \sqrt{y}$ as $y \rightarrow \infty$, and thus satisfies the sublinear growth condition.

6.1. The Ritz method. We can use the Ritz method described in Gelfand and Fomin [10] to provide an approximate numerical solution to this problem in terms of $\psi$, by considering a $\psi=\alpha_{0} \varphi_{0}+\cdots+\alpha_{n} \varphi_{n}$, where $\varphi_{0}, \varphi_{2}, \ldots, \varphi_{n}$ are the first $n+1$ eigenfunctions for the Hilbert space $\mathcal{H}=L^{2}\left(\mathbb{R}, \mu_{\infty}\right)$ of square integrable functions with respect to $\mu_{\infty}(y)$, which can be computed in closed form as $\varphi_{n}(y)=H_{n}(\sqrt{\alpha} y)$ (see Section 6.2.1 in Linetsky [22]). We then optimize for the Fourier coefficients $\left(\alpha_{0}, \ldots, \alpha_{n}\right)$ (see Table 1 and Figure 1$)$.

\subsection{Numerical results.}

TABLE 1

Here we have computed the large-time asymptotic implied volatility $\hat{\sigma}_{\infty}(x)$ using the Ritz method, and compared to the answers obtained using Monte Carlo for $t=75 \mathrm{yrs}$ and $t=30 \mathrm{yrs}$

\begin{tabular}{lccc}
\hline $\mathbf{x}$ & $\hat{\boldsymbol{\sigma}}_{\boldsymbol{\infty}}(\boldsymbol{x})$ & Monte Carlo $\boldsymbol{t}=\mathbf{7 5}$ & Monte Carlo $\boldsymbol{t}=\mathbf{3 0}$ \\
\hline 0.0 & 0.858305 & 0.857258 & 0.856479 \\
0.2 & 0.860926 & 0.859900 & 0.859147 \\
0.4 & 0.868463 & 0.867634 & 0.866509 \\
0.6 & 0.880036 & 0.879524 & 0.877567 \\
0.8 & 0.894606 & 0.894606 & 0.891904 \\
\hline
\end{tabular}


7. Stochastic interest rates and jumps. We now consider the following three-factor model for the log stock price $X_{t}$ under $\mathbb{P}$, which incorporates stochastic volatility and a stochastic short rate driven by a CIR square root process:

$$
\left\{\begin{array}{l}
d X_{t}=\left(r_{t}-\frac{1}{2} \sigma\left(Y_{t}\right)^{2}\right) d t+\sigma\left(Y_{t}\right)\left(\rho d W_{t}^{2}+\bar{\rho} d W_{t}^{1}\right)+d Z_{t} \\
d Y_{t}=\left(-\alpha Y_{t}+g\left(Y_{t}\right)\right) d t+d W_{t}^{2} \\
d r_{t}=\kappa_{r}\left(\theta_{r}-r_{t}\right) d t+\sigma_{r} \sqrt{r_{t}} d W_{t}^{3}
\end{array}\right.
$$

where $W^{1}, W^{2}, W^{3}$ are independent Brownian motions, $x_{0}, \kappa_{r}, \theta_{r}, \sigma_{r}>0,|\rho|<1$ and $2 \kappa_{r} \theta_{r}>\sigma_{r}^{2},{ }^{4}$ and $Z_{t}$ is a Lévy process independent of $W^{1}, W^{2}, W^{3}$ such that $e^{Z_{t}}$ is a martingale, with cumulant generating function (cgf) $V_{J}(p)$ so that $\mathbb{E}\left(e^{p Z_{t}}\right)=e^{V_{J}(p) t}$, and $g, \sigma$ satisfy Assumptions 3.1 and 3.2.

Assume that $V_{J}^{\prime \prime}(p)>0$ and $V_{J}(p)$ is essentially smooth on some interval $\left(p_{-}, p_{+}\right)$[i.e., $\left|V_{J}^{\prime}(p)\right| \rightarrow \infty$ as $p \nearrow p_{+}$and $p \searrow p_{-}$] with $p_{-}<0<1<p_{+}$. If we let $x_{-}=V_{J}^{\prime}(0)$ and $V_{J}^{*}(x)=\sup _{p}\left[p x-V_{J}(p)\right]$ denote the Legendre transform of $V_{J}$, then by the Gärtner-Ellis theorem, $Z_{t} / t$ satisfies the LDP with rate function $V_{J}^{*}(x)$, and $x_{-}$is the unique minimum of $V_{J}^{*}(x)$ where $V_{J}^{*}\left(x_{-}\right)=0$ (see [9] for details).

We will need the following result.

LEMMA 7.1. For the model in (7.1), $\Gamma_{t}=\frac{1}{t} \int_{0}^{t} r_{s} d s$ satisfies the LDP as $t \rightarrow$ $\infty$ with good rate function given by the Fenchel-Legendre transform of $V_{\mathrm{CIR}}$ :

$$
I_{\mathrm{CIR}}(a)=\sup _{a>0}\left\{p a-V_{\mathrm{CIR}}(p)\right\}=\frac{\kappa_{r}^{2}\left(a-\theta_{r}\right)^{2}}{2 a \sigma_{r}^{2}},
$$

where

$$
\begin{aligned}
V_{\mathrm{CIR}}(p) & =\lim _{t \rightarrow \infty} \frac{1}{t} \log \mathbb{E}\left(e^{p \int_{0}^{t} r_{s} d s}\right) \\
& = \begin{cases}\frac{\kappa_{r} \theta_{r}}{\sigma_{r}^{2}}\left[\kappa_{r}-\sqrt{\kappa_{r}^{2}-2 \sigma_{r}^{2} p}\right], & \text { for } p \in\left(-\infty, p_{+}\right], \\
\infty, & \text { for } p \notin\left(-\infty, p_{+}\right],\end{cases}
\end{aligned}
$$

and $p_{+}=\frac{\kappa_{r}^{2}}{2 \sigma_{r}^{2}} . I_{\mathrm{CIR}}$ clearly attains its minimum value of zero at $a=\theta_{r}$.

PROOF. Just follows from the known closed-form expression for the moment generating function of $\Gamma_{t}$ given in, for example, Section 3 in [2] and the GärtnerEllis theorem from large deviations theory, using a similar argument to Theorem 2.1 in Forde and Jacquier [13].

From the contraction principle, we now have the following.

\footnotetext{
${ }^{4}$ Which ensures that $r=0$ is an unattainable boundary.
} 
COROLlary 7.1. $X_{t} / t$ satisfies the LDP as $t \rightarrow \infty$ with rate function $I_{r}(x)=\inf _{a, y, z: a+y+z=x}\left[I(y)+I_{\mathrm{CIR}}(a)+V_{J}^{*}(z)\right]=\inf _{a, y}\left[I(y)+I_{\mathrm{CIR}}(a)+\right.$ $\left.V_{J}^{*}(x-a-y)\right]$, where $I(x)$ is defined as in Theorem 4.1 .

REMARK 7.2. For the model in (7.1), if there is no Lévy process component, by conditioning on $\Gamma_{t}=\frac{1}{t} \int_{0}^{t} r_{s} d s$, we can prove the following asymptotic behaviour for the price of a digital call option in the large-time, large log-moneyness regime:

$$
\lim _{t \rightarrow \infty} \frac{1}{t} \log \mathbb{E}\left(e^{-\int_{0}^{t} r_{s} d s} 1_{X_{t}>x t}\right)=-\inf _{y>x} I_{r}(y),
$$

where $I_{r}(x)=\inf _{a \in \mathbb{R}^{+}}\left[a+I(x-a)+I_{\mathrm{CIR}}(a)\right]$.

REMARK 7.3. We can adapt this result to compute, for example, large-time asymptotics for European call options.

\section{APPENDIX A: LINEAR FUNCTIONALS OF THE OCCUPATION MEASURE}

LEMMA A.1. Consider a linear functional $\Lambda: \mathcal{P}(\mathbb{R}) \mapsto \mathbb{R}$ defined by $\Lambda(\mu)=$ $\int_{-\infty}^{\infty} \lambda(y) \mu(d y)$, where $\lambda$ satisfies the growth condition:

$$
|\lambda(y)| \leq A\left(1+|y|^{q}\right)
$$

for $q \in(0,2), A>0$. Then

$$
\left|\Lambda(\mu)-\Lambda^{m}(\mu)\right| \leq 2 A\left(\frac{1}{m^{2}}+\frac{1}{m^{2-q}}\right)\left(K_{2}(\alpha) I_{Y}(\mu)+K_{3}(\alpha)\right),
$$

where $\Lambda^{m}(\mu)=\int\left[\lambda(y) 1_{\{|y| \leq m\}}+\lambda(m) 1_{\{y>m\}}+\lambda(-m) 1_{\{y<-m\}}\right] \mu(d y)$ and $K_{2}(\alpha)>0$ and $K_{3}(\alpha)$ are the constants introduced in Lemma 2.2.

PROOF. For $I_{Y}(\mu) \leq c$, using the growth condition on $\lambda$ we obtain

$$
\begin{aligned}
\mid \Lambda(\mu) & -\Lambda^{m}(\mu) \mid \\
= & \int_{|y|>m}\left[(\lambda(y)-\lambda(m)) 1_{\{y>m\}}+(\lambda(y)-\lambda(-m)) 1_{\{y<-m\}}\right] \mu(d y) \\
& \leq \int_{|y|>m}\left[(|\lambda(y)|+|\lambda(m)|) 1_{\{y>m\}}+(|\lambda(y)|+|\lambda(-m)|) 1_{\{y<-m\}}\right] \mu(d y) \\
& \leq 4 \int_{|y|>m} A\left(1+|y|^{q}\right) \mu(d y) \\
& \leq 4 A\left(\frac{1}{m^{2}}+\frac{1}{m^{2-q}}\right) \int_{-\infty}^{\infty} y^{2} \mu(d y) \\
& \leq 4 A\left(\frac{1}{m^{2}}+\frac{1}{m^{2-q}}\right)\left(K_{2}(\alpha) I_{Y}(\mu)+K_{3}(\alpha)\right),
\end{aligned}
$$

where we have used Lemma 2.2 in the final line. 
LEMMA A.2. $\sigma^{2}(y)$ satisfies the sub-quadratic growth condition

$$
\sigma^{2}(y) \leq A_{1}\left(1+|y|^{2 p}\right),
$$

where $A_{1}=3 K_{1}^{2}$; thus $F$ [as defined in (3.5)] satisfies the conditions of Lemma A.1 with $\lambda(y)=\sigma^{2}(y), A=A_{1}$ and $q=2 p \in(0,2)$.

PROOF. From the sublinear growth condition $\sigma(y) \leq K_{1}\left(1+|y|^{p}\right)$, we see that

$$
\sigma(y)^{2} \leq A^{2}\left(1+|y|^{p}\right)^{2}=A^{2}\left(1+2|y|^{p}+|y|^{2 p}\right) \leq 3 A^{2}\left(1+|y|^{2 p}\right),
$$

where the final inequality just comes from the inequality $|y|^{p} \leq 1+|y|^{2 p}$.

LEMMA A.3. $b$ satisfies the growth condition

$$
|b(y)| \leq A_{2}\left(1+|y|^{1+p}\right)
$$

for some $A_{2}>0$; hence the functional $G$ defined in Theorem 4.1 satisfies the conditions in Lemma A.1 with $\lambda(y)=b(y), A=A_{2}$ and $q=1+p \in(0,2)$.

PROOF. Using the sublinear growth condition (3.2) and the boundedness of $\sigma^{\prime}$, we see that

$$
\begin{aligned}
|b(y)| & \leq \alpha|y| K_{1}\left(1+|y|^{p}\right)+\frac{1}{2}\left\|\sigma^{\prime}\right\|=\alpha K_{1}|y|+\alpha K_{1}|y|^{1+p}+\frac{1}{2}\left\|\sigma^{\prime}\right\| \\
& \leq \alpha K_{1}\left(1+|y|^{1+p}\right)+\alpha K_{1}|y|^{1+p}+\frac{1}{2}\left\|\sigma^{\prime}\right\| \\
& \leq A_{2}\left(1+|y|^{1+p}\right)
\end{aligned}
$$

for some $A_{2}>0$.

LEMMA A.4. Let $m(y)=-\frac{1}{2} \sigma^{2}(y)+\rho b(y)$. Then $m$ satisfies the growth condition

$$
|m(y)| \leq A_{3}\left(1+|y|^{1+p}\right)
$$

for some $A_{3}>0$; thus $M$ satisfies the conditions in Lemma A.1 with $\lambda(y)=$ $m(y), A=A_{3}$ and $q=1+p \in(0,2)$.

PROOF. Using (A.2) and (A.3),

$$
\begin{aligned}
m(y) & =\left|-\frac{1}{2} \sigma^{2}(y)+\rho b(y)\right| \leq \frac{1}{2} A_{1}\left(1+|y|^{2 p}\right)+\rho A_{2}\left(1+|y|^{1+p}\right) \\
& \leq A_{3}\left(1+|y|^{1+p}\right)
\end{aligned}
$$

for some $A_{3}>0$. 


\section{APPENDIX B: PROOF OF LEMMA 2.1}

To verify the lower bound conditions I and II, we have to show that $p(1, x, d y)$ admits a density $p(1, x, y)$ and that

$$
\lim _{x_{2} \rightarrow x_{1}} \int_{-\infty}^{\infty}\left|p\left(1, x_{2}, y\right)-p\left(1, x_{1}, y\right)\right| d y=0 .
$$

For the rest of the proof, we assume that $Y_{0}=x$. Let $\bar{G}(y)=\int_{x}^{y} g(u) d u$; then $\bar{G}$ has sub-quadratic growth and recall that $\left|g^{\prime}\right|$ is bounded by assumption. Let $h(y):=-\frac{\alpha}{2} y^{2}+\bar{G}(y)$. Then the perturbed OU process $Y$ in (2.4) satisfies $d Y_{t}=$ $h^{\prime}\left(Y_{t}\right) d t+d W_{t}$ and

$$
d h\left(Y_{t}\right)=h^{\prime}\left(Y_{t}\right)\left(h^{\prime}\left(Y_{t}\right) d t+d W_{t}\right)+\frac{1}{2} h^{\prime \prime}\left(Y_{t}\right) d t
$$

We now define a measure $\mathbb{Q}$ such that

$$
\left.\frac{d \mathbb{Q}}{d \mathbb{P}}\right|_{\mathcal{F}_{t}}:=e^{-\frac{1}{2} \int_{0}^{t} h^{\prime}\left(Y_{s}\right)^{2} d s-\int_{0}^{t} h^{\prime}\left(Y_{s}\right) d W_{s}}=e^{h(x)-h\left(Y_{t}\right)+\frac{1}{2} \int_{0}^{t} \tilde{g}\left(Y_{s}\right) d s},
$$

where $\tilde{g}(y)=h^{\prime \prime}(y)+\left(h^{\prime}(y)\right)^{2}=\left(-\alpha+g^{\prime}(y)\right)+(-\alpha y+g(y))^{2}$ and we have used (B.2) to remove the stochastic integral term in (B.3). To check that the righthand side in (B.3) is a $\mathbb{P}$-martingale, we first define an intermediate change of measure $\left.\frac{d \mathbb{P}^{\mathrm{OU}}}{d \mathbb{P}}\right|_{\mathcal{F}_{t}}:=M_{1}(t)$, where

$$
M_{1}(t)=e^{-\frac{1}{2} \int_{0}^{t} g^{2}\left(Y_{s}\right) d s-\int_{0}^{t} g\left(Y_{s}\right) d W_{s}} .
$$

Then $M_{1}$ is a $\mathbb{P}$-martingale since the Novikov condition is satisfied following the same argument as Appendix D, and

$$
d Y_{t}=-\alpha Y_{t} d t+d \tilde{W}_{t},
$$

where $\tilde{W}_{t}=W_{t}-\int_{0}^{t} g\left(Y_{s}\right) d s$ is a Brownian motion under $\mathbb{P}^{\mathrm{OU}}$, that is, $Y$ is an (unperturbed) OU process under $\mathbb{P}^{\mathrm{OU}}$. Now define $\left.\frac{d \mathbb{Q}}{d \mathbb{P O U}}\right|_{\mathcal{F}_{t}}:=M_{2}(t)$, where

$$
M_{2}(t)=e^{-\frac{1}{2} \int_{0}^{t}\left(\alpha^{2} Y_{s}^{2}\right) d s-\int_{0}^{t} \alpha Y_{s} d \tilde{W}_{s}} .
$$

If $M_{2}(t)$ is a $\mathbb{P}^{\mathrm{OU}}$-martingale, then we can go straight from $\mathbb{P}$ to $\mathbb{Q}$ and define as in (B.3):

$$
\left.\frac{d \mathbb{Q}}{d \mathbb{P}}\right|_{\mathcal{F}_{t}}:=e^{-\frac{1}{2} \int_{0}^{t} h^{\prime}\left(Y_{s}\right)^{2} d s-\int_{0}^{t} h^{\prime}\left(Y_{s}\right) d W_{s}^{(2)}}=M_{1}(t) M_{2}(t)
$$

and $M_{1} M_{2}$ will be a $\mathbb{P}$-martingale. To check that $M_{2}$ is a $\mathbb{P}^{\mathrm{OU}}$ martingale, we verify the Novikov condition

$$
\begin{aligned}
\mathbb{E}^{\mathbb{P}^{\mathrm{OU}}}\left[e^{\frac{1}{2} \int_{s}^{s+\varepsilon}\left(\alpha^{2} Y_{u}^{2}\right) d u}\right] & =\mathbb{E}^{\mathbb{P}^{\mathrm{OU}}}\left[e^{\frac{1}{2} \frac{1}{\varepsilon} \int_{s}^{s+\varepsilon} \varepsilon \alpha^{2} Y_{u}^{2} d u}\right] \\
& \leq \frac{1}{\varepsilon} \int_{s}^{s+\varepsilon} \mathbb{E}^{\mathbb{P}^{\mathrm{OU}}}\left[e^{\frac{1}{2} \varepsilon \alpha^{2} Y_{u}^{2}}\right] d u
\end{aligned}
$$


by Jensen's inequality. Under $\mathbb{P}^{\mathrm{OU}}, Y_{u} \sim N\left(y_{0} e^{-\alpha u}, \frac{1-e^{-2 \alpha u}}{2 \alpha}\right)$, so taking $\varepsilon$ small enough (say $\varepsilon=\frac{1}{4 \alpha}$ ), we get $\mathbb{E}^{\mathbb{P}^{\mathrm{OU}}}\left[e^{\frac{1}{2} \int_{s}^{s+\varepsilon}\left(\alpha^{2} Y_{u}^{2}\right) d u}\right]<\infty$, for any $s>0$. Thus, by Corollary 5.5.14 on page 199 in [20], $M_{2}$ is a $\mathbb{P}^{\mathrm{OU}}$-martingale.

By Girsanov's theorem, $Y$ is standard Brownian motion under $\mathbb{Q}$ and for any $f \in C_{b}(\mathbb{R})$,

$$
\begin{aligned}
\int_{-\infty}^{\infty} f(y) p(t, x, d y) & =\mathbb{E}^{\mathbb{P}}\left(f\left(Y_{t}\right)\right)=\mathbb{E}^{\mathbb{Q}}\left[f\left(Y_{t}\right) e^{h\left(Y_{t}\right)-h(x)-\frac{1}{2} \int_{0}^{t} \tilde{g}\left(Y_{s}\right) d s}\right] \\
& =\int_{-\infty}^{\infty} f(y) e^{h(y)-h(x)} \mathbb{E}^{\mathbb{Q}}\left(e^{-\frac{1}{2} \int_{0}^{t} \tilde{g}\left(Y_{s}\right) d s} \mid Y_{t}=y\right) \gamma(t, x, y) d y \\
& =\int_{-\infty}^{\infty} f(y) e^{h(y)-h(x)} \phi(t, x, y) \gamma(t, x, y) d y
\end{aligned}
$$

(see also equations (6)-(8) in [24]), where $\gamma(t, x, y)=\frac{1}{\sqrt{2 \pi t}} e^{-(y-x)^{2} / 2 t}, h(y)=$ $\int_{x}^{y}(-\alpha u+g(u)) d u=-\frac{\alpha}{2} y^{2}+\bar{G}(y)+\frac{\alpha}{2} x^{2}-\bar{G}(x)$ and $\bar{G}(y)=\int_{x}^{y} g(u) d u$ and

$$
\phi(t, x, y)=\mathbb{E}^{\hat{\mathbb{P}}_{x, y}}\left(e^{-\frac{1}{2} \int_{0}^{t} \tilde{g}\left(Y_{s}\right) d s}\right),
$$

where $\hat{\mathbb{P}}_{x, y}$ is a probability measure under which $Y$ is a Brownian bridge with $Y_{0}=x$ and $Y_{t}=y$. Thus, $Y$ has a transition density given by

$$
p(t, x, y)=\gamma(t, x, y) e^{h(y)-h(x)} \phi(t, x, y) .
$$

$\bar{G}(y)$ is sub-quadratic so we can choose a constant $c>0$ such that $\bar{G}(y) \leq c+\frac{\alpha y^{2}}{4}$. Then we see that

$$
e^{h\left(Y_{t}\right)-h(x)}=e^{-\frac{\alpha Y_{t}^{2}}{2}+\bar{G}\left(Y_{t}\right)+\frac{\alpha x^{2}}{2}-\bar{G}(x)} \leq e^{\frac{\alpha x^{2}}{2}-\bar{G}(x)} e^{c-\frac{\alpha Y_{t}^{2}}{4}},
$$

and

$$
\begin{aligned}
\phi(t, x, y) & =\mathbb{E}^{\hat{\mathbb{P}}_{x, y}}\left(e^{-\frac{1}{2} \int_{0}^{t} \tilde{g}\left(Y_{s}\right) d s}\right) \\
& =\mathbb{E}^{\hat{\mathbb{P}}_{x, y}}\left(e^{\int_{0}^{t}\left[-\frac{1}{2}\left(-\alpha Y_{s}+g\left(Y_{s}\right)\right)^{2}-\frac{1}{2}\left(-\alpha+g^{\prime}\left(Y_{s}\right)\right)\right] d s}\right) \\
& \leq e^{\frac{\alpha+\left\|g^{\prime}\right\|}{2} t} .
\end{aligned}
$$

Thus, we have

$$
p(1, x, y) \leq \frac{1}{\sqrt{2 \pi}} e^{\frac{\alpha x^{2}}{2}-\bar{G}(x)} e^{c-\frac{\alpha y^{2}}{4}} e^{\frac{\alpha+\left\|g^{\prime}\right\|}{2}}=C_{1} e^{-\frac{\alpha}{4} y^{2}+C_{2}}
$$

for some constants $C_{1}, C_{2}$ which are independent of $y$. Thus, $\sup _{x \in K} p(1, x, y) \leq$ $c_{1} e^{-c y^{2}}$ for any compact set $K \subset \mathbb{R}$. From the main theorem in [24], we also know that $p(t, x, y)$ is continuous in $x$. Hence, we can apply the dominated convergence theorem to establish (B.1). 


\section{APPENDIX C: PROOF OF LEMMA 4.1}

Using the sublinear growth condition on $\sigma$, we have

$$
\begin{aligned}
|\chi(y)| & =\left|\int_{y_{0}}^{y} \sigma(u) d u\right| \leq\left|\int_{y_{0}}^{y} K_{1}\left(1+|u|^{p}\right)\right| d u \leq K_{1}\left|y-y_{0}\right|+K_{1} \int_{y_{0}}^{y}|u|^{p} d u \\
& \leq K_{1}\left|y-y_{0}\right|+\frac{K_{1}}{1+p}\left(|y|^{1+p}+\left|y_{0}\right|^{1+p}\right) .
\end{aligned}
$$

Thus, $\lim \sup _{|y| \rightarrow \infty} \frac{|\chi(y)|}{|y|^{1+p}} \leq \frac{K_{1}}{1+p}$ which implies that

$$
\liminf _{|y| \rightarrow \infty} \frac{\left|\chi^{-1}(y)\right|}{|y|^{r}} \geq \tilde{K}
$$

for some $\tilde{K}>0$, where $r=\frac{1}{1+p} \in\left(\frac{1}{2}, 1\right)$ [note that $\chi^{-1}(\cdot)$ is well defined because $\left.\chi^{\prime}(y)=\sigma(y)>0\right]$. Then from the analysis in the previous Appendix, we have

$$
\begin{aligned}
\mathbb{P}(\chi & \left.\left(Y_{t}\right)>t v\right) \\
& =\mathbb{E}^{\mathbb{Q}}\left[e^{h\left(Y_{t}\right)-h\left(y_{0}\right)-\frac{1}{2} \int_{0}^{t}\left(h^{\prime}\left(Y_{s}\right)\right)^{2} d s-\frac{1}{2} \int_{0}^{t} h^{\prime \prime}\left(Y_{s}\right) d s} 1_{Y_{t}>\chi^{-1}(t v)}\right] \\
(\mathrm{C} .2) & =\mathbb{E}^{\mathbb{Q}}\left[e^{-\frac{\alpha Y_{t}^{2}}{2}+\bar{G}\left(Y_{t}\right)+\frac{\alpha y_{0}^{2}}{2}-\bar{G}\left(y_{0}\right)-\int_{0}^{t}\left(\frac{1}{2}\left(-\alpha Y_{s}+g\left(Y_{s}\right)\right)^{2}+\frac{1}{2}\left(-\alpha+g^{\prime}\left(Y_{s}\right)\right)\right) d s} 1_{Y_{t}>\chi^{-1}(t v)}\right] \\
& \leq e^{\frac{\alpha y_{0}^{2}}{2}-\bar{G}\left(y_{0}\right)+\frac{\alpha+\left\|g^{\prime}\right\|}{2} t+c-\frac{\alpha\left(\tilde{K}(t v)^{r}\right)^{2}}{4}} \mathbb{Q}\left(Y_{t}>\tilde{K}(t v)^{r}\right) \\
& \leq c_{1} e^{-c_{2} t^{2 r}}
\end{aligned}
$$

for $t$ sufficiently large, for some constants $c_{1}, c_{2}>0$, where we have used (C.1) in the penultimate line and that $Y_{t} \sim N\left(y_{0}, t\right)$ under $\mathbb{Q}$.

\section{APPENDIX D: PROOF OF PROPOSITION 5.1}

To show that $S_{t}=e^{-\frac{1}{2} \int_{0}^{t} \sigma^{2}\left(Y_{s}\right) d s+\int_{0}^{t} \sigma\left(Y_{s}\right)\left(\bar{\rho} d W_{s}^{1}+\rho d W_{s}^{2}\right)}$ is a martingale, by Corollary 5.13, page 199 in [20], it is sufficient to check the Novikov condition:

$$
\mathbb{E}\left(e^{\frac{1}{2} \int_{0}^{t} \sigma^{2}\left(Y_{s}\right) d t}\right)<\infty ; \quad 0 \leq t<\infty .
$$

Fix $0<t<\infty$. Define $u_{n}$ as in the proof of Lemma 2.2. Then, as in the proof of Lemma 2.2, $-\frac{L u_{n}}{u_{n}}(y) \rightarrow c_{0} y^{2}-c_{1} y g(y)-c_{2}$ pointwise as $n \rightarrow \infty$, where $c_{0}>0$. Thus, by Fatou's lemma we have

$$
\begin{aligned}
\int_{-\infty}^{\infty} & \left(c_{0} y^{2}-c_{1} y g(y)-c_{2}\right) \mu_{t}(d y) \\
& \leq \liminf _{n \rightarrow \infty} \int_{-\infty}^{\infty}-\frac{L u_{n}}{u_{n}}(y) \mu_{t}(d y) \quad \text { a.s. }
\end{aligned}
$$


and

$$
\begin{aligned}
\mathbb{E}\left[e^{\left.t \int_{-\infty}^{\infty}\left(c_{0} y^{2}-c_{1} y g(y)-c_{2}\right)\right) \mu_{t}(d y)}\right] & \leq \mathbb{E}\left(e^{t \liminf _{n \rightarrow \infty} \int_{-\infty}^{\infty}-\frac{L u_{n}}{u_{n}}(y) \mu_{t}(d y)}\right) \\
& =\mathbb{E}\left(\liminf _{n \rightarrow \infty} e^{t \int_{-\infty}^{\infty}-\frac{L u_{n}}{u_{n}}(y) \mu_{t}(d y)}\right) \\
& \leq \liminf _{n \rightarrow \infty} \mathbb{E}\left[e^{t \int_{-\infty}^{\infty}-\frac{L u_{n}}{u_{n}}(y) \mu_{t}(d y)}\right] .
\end{aligned}
$$

As in the proof of Lemma 2.2, using the sublinear growth of $g$, we can find a constant $C_{1}$ such that $\left.c_{0} y^{2}-c_{1} y g(y)-c_{2}\right) \geq \frac{c_{0}}{2} y^{2}-C_{1}$. From this, we see that

$$
\mathbb{E}\left[e^{-C_{1} t} e^{\frac{1}{2} c_{0} \int_{0}^{t} Y_{s}^{2} d s}\right] \leq \liminf _{n \rightarrow \infty} \mathbb{E}\left[e^{t \int_{-\infty}^{\infty}-\frac{L u_{n}}{u_{n}}(y) \mu_{t}(d y)}\right] .
$$

The right-hand side in (D.1) can be bounded as

$$
\begin{aligned}
\mathbb{E}\left[e^{-\int_{0}^{t} \frac{L u_{n}}{u_{n}}\left(Y_{s}\right) d s}\right] & \leq e^{\log u_{n}\left(Y_{0}\right)} \mathbb{E}\left[e^{\log u_{n}\left(Y_{t}\right)-\log u_{n}\left(Y_{0}\right)-\int_{0}^{t} \frac{L u_{n}}{u_{n}}\left(Y_{s}\right) d s}\right] \\
& \leq u_{n}\left(y_{0}\right),
\end{aligned}
$$

where the inequality follows because $\log u_{n}(y)=\frac{c}{2}\left[n \psi\left(\frac{y}{n}\right)\right]^{2} \geq 0$, and the last equality follows because $M_{t}=e^{\log u_{n}\left(Y_{t}\right)-\log u_{n}\left(Y_{0}\right) \int_{0}^{t} \frac{L u_{n}}{u_{n}}\left(Y_{s}\right) d s}$ is a local martingale with $M_{0}=1$. Applying this to (D.1) and using the definition of $u_{n}(y)$, we get

$$
\mathbb{E}\left[e^{-C_{1} t} e^{\frac{1}{2} c_{0} \int_{0}^{t} Y_{s}^{2} d s}\right] \leq e^{\frac{c}{2} y_{0}^{2}}<\infty .
$$

From Assumption 3.1, we know that $\sigma^{2}(y)$ has sub-quadratic growth, and hence there exists a constant $C_{2}$ such that $\frac{1}{2} \sigma^{2}(y) \leq \frac{c_{0}}{2} y^{2}+C_{2}$. Therefore,

$$
\mathbb{E}\left[e^{\frac{1}{2} \int_{0}^{t} \sigma^{2}\left(Y_{s}\right) d s}\right] \leq \mathbb{E}\left[e^{C_{2} t} e^{\frac{c_{0}}{2} \int_{0}^{t} Y_{s}^{2} d s}\right] \leq e^{\frac{c}{2} y_{0}^{2}+C_{2} t+C_{1} t}<\infty
$$

from (D.3).

Acknowledgments. The authors would like to thank Jin Feng, Lingfei Li, Vadim Linetsky, Matt Lorig, Josef Teichmann, Srinivasa Varadhan and Guanlin Zhang for helpful discussions.

\section{REFERENCES}

[1] Aliprantis, C. D. and Border, K. C. (2006). Infinite Dimensional Analysis: A Hitchhiker's Guide, 3rd ed. Springer, Berlin. MR2378491

[2] Carr, P., Geman, H., Madan, D. B. and Yor, M. (2003). Stochastic volatility for Lévy processes. Math. Finance 13 345-382. MR1995283

[3] Dembo, A. and Zeitouni, O. (1998). Large Deviations Techniques and Applications, 2nd ed. Applications of Mathematics (New York) 38. Springer, New York. MR1619036

[4] Donsker, M. D. and VARAdhan, S. R. S. (1975). Asymptotic evaluation of certain Markov process expectations for large time. I. Comm. Pure Appl. Math. 28 1-47. MR0386024 
[5] Donsker, M. D. and Varadhan, S. R. S. (1976). Asymptotic evaluation of certain Markov process expectations for large time. III. Comm. Pure Appl. Math. 29 389-461. MR0428471

[6] Donsker, M. D. and Varadhan, S. R. S. (1983). Asymptotic evaluation of certain Markov process expectations for large time. IV. Comm. Pure Appl. Math. 36 183-212. MR0690656

[7] Ethier, S. N. and KurTz, T. G. (1986). Markov Processes: Characterization and Convergence. Wiley, New York. MR0838085

[8] Feng, J., Fouque, J.-P. and Kumar, R. (2012). Small-time asymptotics for fast meanreverting stochastic volatility models. Ann. Appl. Probab. 22 1541-1575. MR2985169

[9] FigueroA-López, J., Forde, M. and JACQuier, A. (2011). The large-time smile and skew for exponential Lévy models. Working paper.

[10] Fomin, S. V. and Gelfand, I. M. (2000). Calculus of Variations. Dover, New York.

[11] Forde, M. (2011). Large-time asymptotics for an uncorrelated stochastic volatility model. Statist. Probab. Lett. 81 1230-1232. MR2803767

[12] Forde, M. (2014). The large-maturity smile for the Stein-Stein model. Statist. Probab. Lett. 91 145-152. MR3208128

[13] FORDE, M. and JACQUIER, A. (2011). The large-maturity smile for the Heston model. Finance Stoch. 15 755-780. MR2863641

[14] Forde, M., Jacquier, A. and Mijatović, A. (2010). Asymptotic formulae for implied volatility in the Heston model. Proc. R. Soc. Lond. Ser. A Math. Phys. Eng. Sci. 466 3593-3620. MR2734777

[15] Forde, M. and Zhang, H. Asymptotics for rough stochastic volatility and Lévy models. Preprint.

[16] Friz, P. K. and Victoir, N. B. (2010). Multidimensional Stochastic Processes as Rough Paths: Theory and Applications. Cambridge Studies in Advanced Mathematics 120. Cambridge Univ. Press, Cambridge. MR2604669

[17] Gatheral, J. and JACQuier, A. (2011). Convergence of Heston to SVI. Quant. Finance 11 1129-1132. MR2823299

[18] Jacquier, A., Keller-Ressel, M. and Mijatović, A. (2013). Large deviations and stochastic volatility with jumps: Asymptotic implied volatility for affine models. Stochastics 85 321-345. MR3056193

[19] Jacquier, A., Keller-Ressel, M. and Mijatović, A. (2014). Large deviations for the extended Heston model: The large-time case. Asia-Pac. Financ. Mark. 21 263-280.

[20] Karatzas, I. and Shreve, S. E. (1991). Brownian Motion and Stochastic Calculus, 2nd ed. Graduate Texts in Mathematics 113. Springer, New York. MR1121940

[21] Kontoyiannis, I. and Meyn, S. P. (2005). Large deviations asymptotics and the spectral theory of multiplicatively regular Markov processes. Electron. J. Probab. 10 61-123 (electronic). MR2120240

[22] Linetsky, V. (2004). The spectral decomposition of the option value. Int. J. Theor. Appl. Finance 7 337-384. MR2064020

[23] Pinsky, R. (1985). On evaluating the Donsker-Varadhan I-function. Ann. Probab. 13342 362. MR0781409

[24] Rogers, L. C. G. (1985). Smooth transition densities for one-dimensional diffusions. Bull. Lond. Math. Soc. 17 157-161. MR0806242

[25] Stroock, D. W. (1984). An Introduction to the Theory of Large Deviations. Springer, New York. MR0755154 
[26] VARAdhan, S. R. S. (1984). Large Deviations and Applications. CBMS-NSF Regional Conference Series in Applied Mathematics 46. SIAM, Philadelphia, PA. MR0758258

DEPARTMENT OF MATHEMATICS

KING'S COLLEGE LONDON

STRAND

LONDON, WC2R 2LS

UNITED KINGDOM

E-MAIL: martin.forde@kcl.ac.uk
DEPARTMENT OF MATHEMATICS

WAYNE STATE UNIVERSITY

1243 FACULTY/ADMINISTRATION BUILDING 656 W. KIRBY

DETROIT, MiCHIGAN 48202

USA

E-MAIL: rkumar@math.wayne.edu 